\title{
Global Stabilization of Two Dimensional Viscous Burgers' Equation by Nonlinear Neumann Boundary Feedback Control and Its Finite Element Analysis
}

\author{
Sudeep Kundu ${ }^{1}$ (D) Amiya Kumar Pani ${ }^{2}$
}

Received: 12 September 2019 / Revised: 15 July 2020 / Accepted: 10 August 2020 /

Published online: 24 August 2020

(c) The Author(s) 2020

\begin{abstract}
In this article, global stabilization results for the two dimensional viscous Burgers' equation, that is, convergence of unsteady solution to its constant steady state solution with any initial data, are established using a nonlinear Neumann boundary feedback control law. Then, applying $C^{0}$-conforming finite element method in spatial direction, optimal error estimates in $L^{\infty}\left(L^{2}\right)$ and in $L^{\infty}\left(H^{1}\right)$-norms for the state variable and convergence result for the boundary feedback control law are derived. All the results preserve exponential stabilization property. Finally, several numerical experiments are conducted to confirm our theoretical findings.
\end{abstract}

Keywords 2D-viscous Burgers' equation · Boundary feedback control · Constant steady state $\cdot$ Stabilization $\cdot$ Finite element method $\cdot$ Error estimate $\cdot$ Numerical experiments

Mathematics Subject Classification 35B37 $\cdot 65 \mathrm{M} 60 \cdot 65 \mathrm{M} 15 \cdot 93 \mathrm{~B} 52 \cdot 93 \mathrm{D} 15$

\section{Introduction}

We consider the following Neumann boundary control problem for the two-dimensional viscous Burgers' or Bateman-Burgers equation: seek $u=u(x, t), t>0$ which satisfies

$$
\begin{aligned}
& u_{t}-v \Delta u+u(\nabla u \cdot \mathbf{1})=0 \quad \text { in } \quad(x, t) \in \Omega \times(0, \infty), \\
& \frac{\partial u}{\partial n}(x, t)=v_{2}(x, t) \quad \text { on } \quad(x, t) \in \partial \Omega \times(0, \infty), \\
& u(x, 0)=u_{0}(x) \quad \text { in } \quad x \in \Omega,
\end{aligned}
$$

Sudeep Kundu

sudeep.kundu@uni-graz.at

Amiya Kumar Pani

akp@math.iitb.ac.in

1 Institute of Mathematics and Scientific Computing, University of Graz, Heinrichstr. 36, 8010 Graz, Austria

2 Department of Mathematics, IIT Bombay, Powai, Mumbai 400076, India 
where $\Omega \subset \mathbb{R}^{2}$ is a bounded domain with smooth boundary $\partial \Omega, v>0$ is the diffusion constant with the diffusion term $v \Delta u, v_{2}$ is scalar control input, $\mathbf{1}=(1,1), u(\nabla u \cdot \mathbf{1})=$ $u \sum_{i=1}^{2} u_{x_{i}}$ is the nonlinear convection term and $u_{0}$ is a given function. Application of (1.1) in the area of fluid mechanics is significant to study turbulence behavior, where $u$ is denoted as flow speed of fluid media, $v>0$ is the viscosity parameter which is analogous to the inverse of Reynolds number in the Navier Stokes system. The rate of flow or flux through the boundary is $v_{2}$ which is our control. When $v$ tends to zero in (1.1), it models nonlinear wave propagation.

In literature, several local stabilization results for one dimensional Burgers' equation are available, say for example, see, [5,6] for distributed and Dirichlet boundary control, and [7] for Neumann boundary control under sufficiently smallness assumption on the initial data. We refer to [16,17] and [23] for further references on local stabilization results including results on existence and uniqueness. Related to instantaneous control of 1D Burgers' equation, we refer to [15].

Local stabilization result for 2D viscous Burgers' equation is available in [29] where a nonlinear feedback control law is applied which is obtained through solving HamiltonJacobi-Bellman (HJB) equation and using Riccati based optimal feedback control. For instance, authors first formulate the two dimensional Burgers' equation in abstract form as

$$
w_{t}=A w+F(w)+B v, \quad w(0)=w_{0},
$$

where $A$, with domain $D(A)$, is the infinitesimal generator of an analytic semigroup on a Hilbert space $W, F(w)$ is the nonlinear term, $B$ is the operator from a control space $V$ into $W$. With corresponding cost functional of the form

$$
J\left(w_{v}, v\right)<\infty \text { where } J\left(w_{v}, v\right)=\frac{1}{2} \int_{0}^{\infty}\left(\left\|w_{v}\right\|_{W}^{2}+\|v\|_{V}^{2}\right) d t,
$$

the associated linear feedback control law becomes $v=-B^{*} P w$, where $P$ is the solution to the algebraic Riccati equation for the linear quadratic regulator (LQR) problem. Through solving HJB equation using Taylor series expansion, one can obtain the nonlinear feedback control law as

$$
v=-B^{*} P w+B^{*}\left(A-B B^{*} P\right)^{-*} P F(w),
$$

where $\left(A-B B^{*} P\right)^{-*}$ is the inverse of $\left(A-B B^{*} P\right)^{*}$ (see [29] for more details). Later on, Buchot et al. [4] have discussed local stabilization result in the case of partial information for the two dimensional Burgers' type equation. Subsequently in [25], author has shown local stabilization results for the Navier-Stokes system around a nonconstant steady state solution by constructing a linear feedback control law for the corresponding linearized equation. This, in turn, locally stabilizes the original nonlinear system. All the above mentioned stabilization results are local in nature and are valid under smallness assumption on the data.

Our attempt in this paper is to establish global stabilization result without smallness assumption on the data through the nonlinear Neumann control law using Lyapunov type functional. Such global stabilization results for one dimensional Burgers' equation was earlier studied in $[3,18]$ for both Dirichlet and Neumann boundary control laws. When the coefficient of viscosity is unknown, an adaptive control for one dimensional Burgers' equation is discussed in $[22,26,27]$. Although, effect of these control laws to their state are shown computationally using finite difference method and Chebychev collocation method, but convergence of numerical solution posses some serious difficulty because of the typical nonlinearity present in the system through nonlinear feedback laws. Also in [5,6], authors 
have considered finite element method to solve numerically local stabilization problem for 1D Burgers' equation without any convergence analysis. Subsequently in [19], optimal error estimates in the context of finite element method for the state variable and superconvergence result for the feedback control laws are derived. For related analysis on Benjamin Bona Mahony Burgers' (BBM-Burgers') type equations, we refer to [20]. Concerning extensive literature for one dimensional Burgers' and BBM-Burgers' problem, see the references in $[19,20]$.

To the best of our knowledge, there is hardly any result on global stabilization for the two dimensional Burgers' equation. Further to continue our investigation keeping an eye on the Navier-Stokes system, finite element method is applied to 2D Burgers' equation, that is, the Eq. (1.1).

The major contributions of this article are summarized as follows:

- With the help of Lyapunov functional, a nonlinear Neumann feedback control law for the problem (1.1)-(1.3) is derived and global stabilization results in $L^{\infty}\left(H^{i}\right)(i=0,1,2)$ norms are established.

- Based on $C^{0}$-conforming finite element method in spatial direction, optimal error estimates, (optimality with respect to approximation property) for the state variable and for the feedback control law are derived keeping time variable continuous.

- Several numerical examples including an example in which a part of boundary is with Neumann control and other part is with Dirichlet boundary condition are given to illustrate our theoretical findings.

For the rest of the article, denote $H^{m}(\Omega)=W^{m, 2}(\Omega)$ to be the standard Sobolev space with norm $\|\cdot\|_{m}$, and seminorm $|\cdot|_{m}$. For $m=0$, it corresponds to the usual $L^{2}$ norm and is denoted by $\|\cdot\|$. The space $L^{p}((0, T) ; X) 1 \leq p \leq \infty$, consists of all strongly measurable functions $v:[0, T] \rightarrow X$ with norm

$$
\|v\|_{L^{p}((0, T) ; X)}:=\left(\int_{0}^{T}\|v(t)\|_{X}^{p} d t\right)^{\frac{1}{p}}<\infty \text { for } 1 \leq p<\infty,
$$

and

$$
\|v\|_{L^{\infty}((0, T) ; X)}:=\underset{0 \leq t \leq T}{\operatorname{ess} \sup }\|v(t)\|_{X}<\infty .
$$

The rest of the paper is organized as follows. While Sect. 2 is on problem formulation and preliminaries, Sect. 3 focuses on global stabilization results using a nonlinear feedback control law. Section 4 deals with finite element approximation for the semidiscrete system. Further, optimal error estimates are obtained for the state variable and convergence result is derived for the feedback control law. Finally, Sect. 5 concludes with some numerical experiments.

\section{Preliminaries and Problem Formulation}

This section focuses on some preliminary results to be used in our subsequent sections. Further, it deals with the Neumann control law using Lyapunov functional and with problem formulation for our global stabilizability and finite element analysis on our latter sections.

The following trace embedding result holds for 2D.

Boundary Trace Imbedding Theorem (p. 164, [1]): There exists a bounded linear map

$$
T: H^{1}(\Omega) \hookrightarrow L^{q}(\partial \Omega) \text { for } 2 \leq q<\infty
$$


such that

$$
\|T y\|_{L^{q}(\partial \Omega)} \leq C\|y\|_{H^{1}(\Omega)},
$$

for each $y \in H^{1}(\Omega)$, with the constant $C$ depend on $q$ and $\Omega$. Also the following trace result holds

\section{Trace inequality [21]:}

$$
\|T y\|_{H^{s}(\partial \Omega)} \leq C\|y\|_{H^{s+\frac{1}{2}(\Omega)}}, \quad s \neq 1, \quad 0<s \leq \frac{3}{2} .
$$

Below, we recall the following inequalities for our subsequent use:

Friedrichs's inequality: For $y \in H^{1}(\Omega)$, there holds

$$
\|y\|^{2} \leq C_{F}\left(\|\nabla y\|^{2}+\|y\|_{L^{2}(\partial \Omega)}^{2}\right),
$$

where $C_{F}>0$ is the Friedrichs's constant.

More precisely, in 2D we have

$$
\int_{\Omega} y^{2} d x=\int_{\Omega} y^{2} \Delta \phi d x
$$

where $\phi(x)=\frac{1}{4}|x|^{2}$ so that $\Delta \phi=1$. Now integrate by parts to obtain

$$
\begin{aligned}
\int_{\Omega} y^{2} d x & =-2 \int_{\Omega} y \nabla y \nabla \phi d x+\frac{1}{2} \int_{\partial \Omega} y^{2}(x \cdot n) d \Gamma \\
& =-\int_{\Omega} y \nabla y x d x+\frac{1}{2} \int_{\partial \Omega} y^{2}(x \cdot n) d \Gamma \\
& \leq \frac{1}{2} \int_{\Omega} y^{2} d x+\frac{1}{2} \sup _{x \in \partial \Omega}|x|^{2} \int_{\Omega}|\nabla y|^{2} d x+\frac{1}{2} \sup _{x \in \partial \Omega}|x| \int_{\partial \Omega} y^{2} d \Gamma .
\end{aligned}
$$

Therefore, it follows that

$$
\int_{\Omega} y^{2} d x \leq \sup _{x \in \partial \Omega}|x|^{2} \int_{\Omega}|\nabla y|^{2} d x+\sup _{x \in \partial \Omega}|x| \int_{\partial \Omega} y^{2} d \Gamma .
$$

Hence, the Friedrichs's inequality constant can be taken as $C_{F}=$ $\max \left\{\sup _{x \in \partial \Omega}|x|^{2}, \sup _{x \in \partial \Omega}|x|\right\}$. Gagliardo-Nirenberg inequality (see [24]): For $w \in$ $H^{1}(\Omega)$, we have

$$
\begin{aligned}
\|w\|_{L^{4}} & \leq C\left(\|w\|^{1 / 2}\|\nabla w\|^{1 / 2}+\|w\|\right), \quad \text { and for } w \in H^{2}(\Omega), \quad \text { we have } \\
\|\nabla w\|_{L^{4}} & \leq C\left(\|w\|^{1 / 4}\|\Delta w\|^{3 / 4}+\|w\|\right) .
\end{aligned}
$$

Agmon's inequality (see [2]): For $z \in H^{2}(\Omega)$, there holds

$$
\|z\|_{L^{\infty}} \leq C\left(\|z\|^{\frac{1}{2}}\|\Delta z\|^{\frac{1}{2}}+\|z\|\right) .
$$

Now the corresponding equilibrium or steady state problem of (1.1)-(1.3) becomes: find $u^{\infty}$ as a solution of

$$
\begin{aligned}
-v \Delta u^{\infty}+u^{\infty}\left(\nabla u^{\infty} \cdot \mathbf{1}\right) & =0 \quad \text { in } \Omega, \\
\frac{\partial u^{\infty}}{\partial n} & =0 \text { on } \partial \Omega .
\end{aligned}
$$


Note that any constant $w_{d}$ satisfies (2.4)-(2.5). Without loss of generality, we assume that $w_{d} \geq 0$. When $v$ is sufficiently small and initial condition $u_{0}$ is antisymmetric, the numerical solution of (1.1)-(1.3) with $\frac{\partial u}{\partial n}=0$ may converge to a nonconstant steady state solution for which related references are given in [19]. We do not consider such cases here. To achieve

$$
\lim _{t \rightarrow \infty} u(x, t)=w_{d} \quad \forall x \in \Omega,
$$

it is enough to consider $\lim _{t \rightarrow \infty} w=0$, where $w=u-w_{d}$ and $w$ satisfies

$$
\begin{aligned}
& w_{t}-v \Delta w+w_{d}(\nabla w \cdot \mathbf{1})+w(\nabla w \cdot \mathbf{1})=0 \quad \text { in } \quad(x, t) \in \Omega \times(0, \infty), \\
& \frac{\partial w}{\partial n}(x, t)=v_{2}(x, t), \quad \text { on } \partial \Omega \times(0, \infty), \\
& w(0)=u_{0}-w_{d}=w_{0} \text { (say) in } \Omega .
\end{aligned}
$$

The motivation behind choosing the Neumann boundary control comes from the physical situation. Say for example, in thermal problem, one cannot actuate the temperature $w$ on the boundary, but the heat flux $\frac{\partial w}{\partial n}$. This makes the stabilization problem nontrivial because $w_{d}$ is not asymptotically stable with zero Neumann boundary data. Concerning Dirichlet boundary control unlike in 1D [18], it is not easy to get a concrete useful form of the control law. Although the control law $v_{2}$ derived in (2.13), is in invertible form so we can obtain $w$ in terms of $\frac{\partial w}{\partial n}$ on the boundary by solving cubic equation using Cardan's method, but that form is not useful for the stabilizability analysis.

For our analysis, the following compatibility conditions for $w_{0}$ on the boundary are required, namely;

$$
\frac{\partial w_{0}}{\partial n}=v_{2}(x, 0) \text { and } \frac{\partial w_{t}}{\partial n}(x, 0)=v_{2 t}(x, 0),
$$

where $v_{2}(x, \cdot)$ is continuously differentiable at $t=0$ for almost all $x$. These conditions are required for the proof of Lemmas 3.4 and 3.5.

Now, the motivation for choosing the control law comes from the construction of a Lyapunov functional of the following form $V(t)=\frac{1}{2} \int_{\Omega} w(x, t)^{2} d x$. Hence, on taking derivative with respect to time, we arrive at

$$
\begin{aligned}
\frac{d V}{d t} & =\int_{\Omega} w\left(v \Delta w-w_{d}(\nabla w \cdot \mathbf{1})-w(\nabla w \cdot \mathbf{1})\right) d x \\
& =-v\|\nabla w\|^{2}+v \int_{\partial \Omega} \frac{\partial w}{\partial n} w d \Gamma-\int_{\Omega} w_{d}(\nabla w \cdot \mathbf{1}) w d x-\int_{\Omega} w(\nabla w \cdot \mathbf{1}) w d x .
\end{aligned}
$$

Using the Young's inequality, it follows that

$$
\begin{aligned}
w_{d}((\nabla w \cdot \mathbf{1}), w) & =\frac{w_{d}}{2} \int_{\Omega}\left(\left(w^{2}\right)_{x_{1}}+\left(w^{2}\right)_{x_{2}}\right) d x=\frac{w_{d}}{2} \sum_{j=1}^{2} \int_{\partial \Omega} w^{2} \cdot v_{j} d \Gamma \\
& \leq \frac{w_{d}}{\sqrt{2}} \int_{\partial \Omega} w^{2} d \Gamma \leq w_{d} \int_{\partial \Omega} w^{2} d \Gamma
\end{aligned}
$$

and

$$
\begin{aligned}
\int_{\Omega} w(\nabla w \cdot \mathbf{1}) w d x & \leq \frac{1}{3} \sum_{j=1}^{2} \int_{\partial \Omega} w^{3} \cdot v_{j} d \Gamma \leq \frac{1}{3} \sqrt{2} \int_{\partial \Omega}|w|^{3} d \Gamma \\
& \leq c_{0} \int_{\partial \Omega} w^{2} d \Gamma+\frac{1}{18 c_{0}} \int_{\partial \Omega} w^{4} d \Gamma,
\end{aligned}
$$


where $c_{0}$ is a positive constant. Therefore, it follows that

$$
\frac{d V}{d t} \leq-v\|\nabla w\|^{2}+\int_{\partial \Omega}\left(v \frac{\partial w}{\partial n}+\left(w_{d}+c_{0}\right) w+\frac{1}{18 c_{0}} w^{3}\right) w d \Gamma .
$$

Now, choose the Neumann boundary feedback control law as

$$
v_{2}(x, t)=-\frac{1}{v}\left(2\left(c_{0}+w_{d}\right) w+\frac{2}{9 c_{0}} w^{3}\right) \text { on } \partial \Omega,
$$

to obtain

$$
\begin{aligned}
\frac{d V}{d t} & \leq-v\|\nabla w\|^{2}-\left(c_{0}+w_{d}\right) \int_{\partial \Omega} w^{2} d \Gamma-\frac{1}{6 c_{0}} \int_{\partial \Omega} w^{4} d \Gamma \\
& \leq-\min \left\{v,\left(c_{0}+w_{d}\right)\right\}\left(\|\nabla w\|^{2}+\|w\|_{L^{2}(\partial \Omega)}^{2}\right) \\
& \leq-\frac{2}{C_{F}} \min \left\{v,\left(c_{0}+w_{d}\right)\right\}\left(\frac{1}{2}\|w\|^{2}\right) \leq-C_{L y p} V,
\end{aligned}
$$

where $C_{L y p}=\frac{2}{C_{F}} \min \left\{v,\left(c_{0}+w_{d}\right)\right\}>0$.

Setting $B(v ; w, \phi)$ as $B(v ; w, \phi)=(v(\nabla w \cdot \mathbf{1}), \phi), w$ satisfies a weak form of (2.6)-(2.8) as

$$
\begin{aligned}
\left(w_{t}, v\right)+ & v(\nabla w, \nabla v)+w_{d}(\nabla w \cdot \mathbf{1}, v)+B(w ; w, v) \\
+ & \left\langle\left(2 c_{0}+2 w_{d}\right) w+\frac{2}{9 c_{0}} w^{3}, v\right\rangle_{\partial \Omega}=0 \quad \forall v \in H^{1}(\Omega),
\end{aligned}
$$

with $w(0)=w_{0}$, where $\langle v, w\rangle_{\partial \Omega}:=\int_{\partial \Omega} v w d \Gamma$.

For our subsequent analysis, we assume that there exists a unique weak solution $w$ of (2.14) satisfying the following regularity results

$$
\|w(t)\|_{2}^{2}+\left\|w_{t}(t)\right\|_{1}^{2}+\int_{0}^{t}\left\|w_{t}(s)\right\|_{2}^{2} d s \leq C .
$$

For existence and uniqueness with continuous dependence property of one dimensional Burgers' equation with similar type nonlinearity, see, [17,22] and their arguments can be modified to prove the wellposedness of the problem (2.14). For regularity results, the energy method applied in our Sect. 3 can be appropriately modified to prove (2.15). Therefore, we shall not pursue it further in this article.

Throughout the paper $C$ is a generic positive constant.

\section{Stabilization Results}

In this section, we establish global stabilization results of the continuous problem (1.1)-(1.3). More precisely, exponential stabilization results for the state variable $w(t)$ are shown for the modified problem (2.6)-(2.8), where feedback control $v_{2}$ is given in (2.13). Moreover, additional regularity results are established assuming compatibility conditions, which are crucial for proving optimal error estimates for the state variable.

Our results of this section are based on energy arguments using exponential weight functions. For similar analysis, see, [8,11,12,14,28,30]. 
Throughout this section, all the results hold with the same decay rate $\alpha$ :

$$
0 \leq \alpha \leq \frac{1}{C_{F}} \min \left\{v,\left(c_{0}+w_{d}\right)\right\}
$$

Lemma 3.1 Let $w_{0} \in L^{2}(\Omega)$. Then, there holds

$$
\|w(t)\|^{2}+\beta e^{-2 \alpha t} \int_{0}^{t} e^{2 \alpha s}\left(\|\nabla w(s)\|^{2}+\|w(s)\|_{L^{2}(\partial \Omega)}^{2}+\frac{1}{3 \beta c_{0}}\|w(s)\|_{L^{4}(\partial \Omega)}^{4}\right) d s \leq e^{-2 \alpha t}\left\|w_{0}\right\|^{2},
$$

where $\beta=2 \min \left\{\left(v-\alpha C_{F}\right),\left(c_{0}+w_{d}-\alpha C_{F}\right)\right\}>0$, and $C_{F}>0$ is the constant in the Friedrichs's inequality (2.3).

Proof Set $v=e^{2 \alpha t} w$ in (2.14) to obtain

$$
\begin{gathered}
\frac{d}{d t}\left\|e^{\alpha t} w\right\|^{2}-2 \alpha\left\|e^{\alpha t} w\right\|^{2}+2 v\left\|e^{\alpha t} \nabla w\right\|^{2}+2 e^{2 \alpha t} \int_{\partial \Omega}\left(\left(2 c_{0}+2 w_{d}\right) w^{2}+\frac{2}{9 c_{0}} w^{4}\right) d \Gamma \\
=-2 w_{d} e^{2 \alpha t}((\nabla w \cdot \mathbf{1}), w)-2 e^{2 \alpha t} B(w ; w, w) .
\end{gathered}
$$

For the first term on the right hand side of (3.2), we use (2.10) to bound it as

$$
2 w_{d} e^{2 \alpha t}((\nabla w \cdot \mathbf{1}), w) \leq \sqrt{2} w_{d} e^{2 \alpha t} \int_{\partial \Omega} w^{2} d \Gamma \leq 2 w_{d} e^{2 \alpha t} \int_{\partial \Omega} w^{2} d \Gamma
$$

For the second term on the right hand side of (3.2), a use of (2.11) with the Young's inequality yields

$$
2 e^{2 \alpha t} B(w ; w, w) \leq \frac{2}{3} e^{2 \alpha t} \sqrt{2} \int_{\partial \Omega}|w|^{3} d \Gamma \leq 2 c_{0} e^{2 \alpha t} \int_{\partial \Omega} w^{2} d \Gamma+\frac{1}{9 c_{0}} e^{2 \alpha t} \int_{\partial \Omega} w^{4} d \Gamma .
$$

Now, using the Friedrichs's inequality (2.3), it follows that

$$
-2 \alpha e^{2 \alpha t}\|w\|^{2} \geq-2 \alpha e^{2 \alpha t} C_{F}\left(\|\nabla w\|^{2}+\|w\|_{L^{2}(\partial \Omega)}^{2}\right)
$$

Hence, from (3.2), we arrive using (3.3)- (3.5) at

$$
\begin{aligned}
\frac{d}{d t}\left\|e^{\alpha t} w\right\|^{2} & +2\left(v-\alpha C_{F}\right)\left\|e^{\alpha t} \nabla w\right\|^{2}+2 e^{2 \alpha t}\left(\left(c_{0}+w_{d}-\alpha C_{F}\right) \int_{\partial \Omega} w^{2} d \Gamma\right. \\
& \left.+\frac{1}{6 c_{0}} \int_{\partial \Omega} w^{4} d \Gamma\right) \leq 0 .
\end{aligned}
$$

Since decay rate satisfy (3.1), the coefficients on the left hand side of (3.6) are non-negative. Integrate (3.6) with respect to time from 0 to $t$, and then, multiply the resulting inequality by $e^{-2 \alpha t}$ to obtain 


$$
\begin{aligned}
\|w(t)\|^{2}+2\left(v-\alpha C_{F}\right) e^{-2 \alpha t} \int_{0}^{t} e^{2 \alpha s}\|\nabla w(s)\|^{2} d s & +2 e^{-2 \alpha t} \int_{o}^{t} e^{2 \alpha s}\left(\left(c_{0}+w_{d}-\alpha C_{F}\right)\|w(s)\|_{L^{2}(\partial \Omega)}^{2}\right. \\
& \left.+\frac{1}{6 c_{0}}\|w(s)\|_{L^{4}(\partial \Omega)}^{4}\right) d s \leq e^{-2 \alpha t}\left\|w_{0}\right\|^{2} .
\end{aligned}
$$

This completes the proof.

Remark 3.1 The above Lemma also holds for $\alpha=0$, that is,

$$
\|w(t)\|^{2}+2 v \int_{0}^{t}\|\nabla w(s)\|^{2} d s+2 \int_{0}^{t}\left(\int_{\partial \Omega}\left(\left(c_{0}+w_{d}\right) w(s)^{2}+\frac{1}{6 c_{0}} w(s)^{4}\right) d \Gamma\right) d s \leq\left\|w_{0}\right\|^{2} .
$$

Moreover, by the Friedrichs's inequality, it follows that

$$
e^{-2 \alpha t} \int_{0}^{t} e^{2 \alpha s}\|w(s)\|^{2} d s \leq C e^{-2 \alpha t}\left\|w_{0}\right\|^{2} .
$$

Remark 3.2 Now instead of taking the control on the whole boundary, if we take the above mentioned Neumann control on some part of the boundary $\left(\Gamma_{N}\right)$ where $\Gamma_{N}$ has nonzero measure with remaining part zero Dirichlet boundary condition, still the stabilization result holds. For instance, consider $\partial \Omega=\Gamma_{D} \cup \Gamma_{N}$ with $\Gamma_{D} \cap \Gamma_{N}=\phi$, where $\Gamma_{D}$ and $\Gamma_{N}$ are sufficiently smooth. With this setting, from (3.2), we arrive at

$$
\begin{aligned}
\frac{d}{d t}\left\|e^{\alpha t} w\right\|^{2}- & 2 \alpha\left\|e^{\alpha t} w\right\|^{2}+2 v\left\|e^{\alpha t} \nabla w\right\|^{2}+2 e^{2 \alpha t} \int_{\Gamma_{N}}\left(\left(2 c_{0}+2 w_{d}\right) w^{2}+\frac{2}{9 c_{0}} w^{4}\right) d \Gamma \\
= & -2 w_{d} e^{2 \alpha t}((\nabla w \cdot \mathbf{1}), w)-2 e^{2 \alpha t} B(w ; w, w) \\
\leq & 2 w_{d} e^{2 \alpha t} \int_{\Gamma_{N}} w^{2} d \Gamma+2 c_{0} e^{2 \alpha t} \int_{\Gamma_{N}} w^{2} d \Gamma+\frac{1}{9 c_{0}} e^{2 \alpha t} \int_{\Gamma_{N}} w^{4} d \Gamma .
\end{aligned}
$$

Using Friedrichs's inequality $\|v\|^{2} \leq C_{F}\left(\|\nabla v\|^{2}+\|v\|_{L^{2}\left(\Gamma_{N}\right)}^{2}\right)$, we obtain

$$
\begin{aligned}
\frac{d}{d t}\left\|e^{\alpha t} w\right\|^{2} & +2\left(v-\alpha C_{F}\right)\left\|e^{\alpha t} \nabla w\right\|^{2}+2 e^{2 \alpha t}\left(\left(c_{0}+w_{d}-2 \alpha C_{F}\right) \int_{\Gamma_{N}} w^{2} d \Gamma\right. \\
& \left.+\frac{1}{6 c_{0}} \int_{\Gamma_{N}} w^{4} d \Gamma\right) \leq 0 .
\end{aligned}
$$

Proceed as before to complete the rest of the proof for $L^{2}$ - stabilization result. In higher order norm, stabilization result also holds similarly when control works on some part of the boundary.

Lemma 3.2 Let $w_{0} \in H^{1}(\Omega)$. Then, for $C=C\left(\left\|w_{0}\right\|_{1}\right)$ there holds

$$
\begin{aligned}
\left(\|\nabla w(t)\|^{2}\right. & \left.+\frac{2\left(c_{0}+w_{d}\right)}{v}\|w(t)\|_{L^{2}(\partial \Omega)}^{2}+\frac{1}{9 v c_{0}}\|w(t)\|_{L^{4}(\partial \Omega)}^{4}\right)+v e^{-2 \alpha t} \int_{0}^{t}\left\|e^{\alpha s} \Delta w(s)\right\|^{2} d s \\
& \leq C e^{C} e^{-2 \alpha t} .
\end{aligned}
$$

Proof Form an $L^{2}$-inner product between (2.6) and $-e^{2 \alpha t} \Delta w$ to obtain

$$
\begin{gathered}
\frac{d}{d t}\left\|e^{\alpha t} \nabla w\right\|^{2}-2 \alpha e^{2 \alpha t}\|\nabla w\|^{2}+2 v\left\|e^{\alpha t} \Delta w\right\|^{2}+\frac{2}{v} \int_{\partial \Omega} e^{2 \alpha t}\left(2\left(c_{0}+w_{d}\right) w+\frac{2}{9 c_{0}} w^{3}\right) w_{t} d \Gamma \\
=2 e^{2 \alpha t} w_{d}(\nabla w \cdot \mathbf{1}, \Delta w)+2 e^{2 \alpha t} B(w ; w, \Delta w) .
\end{gathered}
$$


The fourth term on the left hand side of (3.9) can be rewritten as

$$
\begin{aligned}
\frac{2}{v} \int_{\partial \Omega} e^{2 \alpha t} & \left(2\left(c_{0}+w_{d}\right) w+\frac{2}{9 c_{0}} w^{3}\right) w_{t} d \Gamma \\
= & \frac{d}{d t}\left(\frac{2\left(c_{0}+w_{d}\right)}{v}\left\|e^{\alpha t} w\right\|_{L^{2}(\partial \Omega)}^{2}+\frac{1}{9 v c_{0}}\left(e^{2 \alpha t}\|w\|_{L^{4}(\partial \Omega)}^{4}\right)\right) \\
& \quad-2 \alpha e^{2 \alpha t}\left(\frac{2\left(c_{0}+w_{d}\right)}{v}\|w\|_{L^{2}(\partial \Omega)}^{2}+\frac{1}{9 v c_{0}}\|w\|_{L^{4}(\partial \Omega)}^{4}\right) .
\end{aligned}
$$

The terms on the right hand side of (3.9) are bounded by

$$
2 e^{2 \alpha t} w_{d}(\nabla w \cdot \mathbf{1}, \Delta w) \leq \frac{v}{2}\left\|e^{\alpha t} \Delta w\right\|^{2}+\frac{2}{v} e^{2 \alpha t} w_{d}^{2}\|\nabla w\|^{2},
$$

and using Gagliardo-Nirenberg inequality and Lemma 3.1, by

$$
\begin{aligned}
2 e^{2 \alpha t} B(w ; w, \Delta w) \leq & C e^{2 \alpha t}\|w\|_{L^{4}}\|\nabla w\|_{L^{4}}\|\Delta w\| \\
\leq & C e^{2 \alpha t}\left(\|w\|^{\frac{1}{2}}\|\nabla w\|^{\frac{1}{2}}+\|w\|\right)\left(\|w\|^{\frac{1}{4}}\|\Delta w\|^{\frac{3}{4}}+\|w\|\right)\|\Delta w\| \\
\leq & C e^{2 \alpha t}\left(\|w\|^{\frac{3}{4}}\|\nabla w\|^{\frac{1}{2}}\|\Delta w\|^{\frac{7}{4}}+\|w\|^{\frac{5}{4}}\|\Delta w\|^{\frac{7}{4}}+\|w\|^{\frac{3}{2}}\|\nabla w\|^{\frac{1}{2}}\|\Delta w\|\right. \\
& \left.\quad+\|w\|^{2}\|\Delta w\|\right) \\
\leq & \frac{v}{2}\left\|e^{\alpha t} \Delta w\right\|^{2}+C e^{2 \alpha t}\|w\|^{2}\|\nabla w\|^{4}+C e^{2 \alpha t}\|w\|^{2}+C e^{2 \alpha t}\|w\|^{2}\|\nabla w\|^{2} .
\end{aligned}
$$

Finally, from (3.9), we arrive at

$$
\begin{aligned}
\frac{d}{d t}\left(e ^ { 2 \alpha t } \left(\|\nabla w\|^{2}+\right.\right. & \left.\left.\frac{2\left(c_{0}+w_{d}\right)}{v}\|w\|_{L^{2}(\partial \Omega)}^{2}+\frac{1}{9 v c_{0}}\|w\|_{L^{4}(\partial \Omega)}^{4}\right)\right)+v\left\|e^{\alpha t} \Delta w\right\|^{2} \\
\leq & 2 \alpha e^{2 \alpha t}\left(\frac{2\left(c_{0}+w_{d}\right)}{v}\|w\|_{L^{2}(\partial \Omega)}^{2}+\frac{1}{9 v c_{0}}\|w\|_{L^{4}(\partial \Omega)}^{4}\right)+\frac{2}{v} e^{2 \alpha t} w_{d}^{2}\|\nabla w\|^{2} \\
& +C e^{2 \alpha t}\|w\|^{2}+C e^{2 \alpha t}\|w\|^{2}\|\nabla w\|^{2}+C e^{2 \alpha t}\|w\|^{2}\|\nabla w\|^{4} .
\end{aligned}
$$

Integrate the above inequality from 0 to $t$, and then use the Grönwall's inequality with Lemma 3.1 to obtain

$$
\begin{aligned}
e^{2 \alpha t}\left(\|\nabla w(t)\|^{2}\right. & \left.+\frac{2\left(c_{0}+w_{d}\right)}{v}\|w(t)\|_{L^{2}(\partial \Omega)}^{2}+\frac{1}{9 v c_{0}}\|w(t)\|_{L^{4}(\partial \Omega)}^{4}\right)+v \int_{0}^{t}\left\|e^{\alpha s} \Delta w(s)\right\|^{2} d s \\
& \leq C\left(\left\|w_{0}\right\|_{1}^{2}+\left\|w_{0}\right\|_{L^{2}(\partial \Omega)}^{2}+\left\|w_{0}\right\|_{L^{4}(\partial \Omega)}^{4}\right) \exp \left(C \int_{0}^{t}\|w\|^{2}\left(1+\|\nabla w\|^{2}\right) d s\right) .
\end{aligned}
$$

Use Remark 3.1 for the integral term under the exponential sign, and then multiply the resulting inequality by $e^{-2 \alpha t}$ to complete the rest of the proof.

Lemma 3.3 Let $w_{0} \in H^{1}(\Omega)$. Then, there exists a positive constant $C=C\left(\left\|w_{0}\right\|_{1}\right)$ such that the following estimate holds.

$$
\begin{gathered}
\left(v\|\nabla w(t)\|^{2}+2\left(c_{0}+w_{d}\right)\|w(t)\|_{L^{2}(\partial \Omega)}^{2}+\frac{1}{9 c_{0}}\|w(t)\|_{L^{4}(\partial \Omega)}^{4}\right) \\
+e^{-2 \alpha t} \int_{0}^{t} e^{2 \alpha s}\left\|w_{t}(s)\right\|^{2} d s \leq C e^{C} e^{-2 \alpha t} .
\end{gathered}
$$


Proof Choose $v=e^{2 \alpha t} w_{t}$ in (2.14) to obtain

$$
\begin{aligned}
2\left\|e^{\alpha t} w_{t}\right\|^{2}+v \frac{d}{d t}\left\|e^{\alpha t} \nabla w\right\|^{2} & -2 v \alpha\left\|e^{\alpha t} \nabla w\right\|^{2}+2 \int_{\partial \Omega}\left(2\left(c_{0}+w_{d}\right) w+\frac{2}{9 c_{0}} w^{3}\right) e^{2 \alpha t} w_{t} d \Gamma \\
& =-2 w_{d} e^{2 \alpha t}\left(\nabla w \cdot \mathbf{1}, w_{t}\right)-2 e^{2 \alpha t} B\left(w ; w, w_{t}\right) .
\end{aligned}
$$

The terms on the right hand side of (3.11) are bounded by

$$
2 w_{d} e^{2 \alpha t}\left(\nabla w \cdot \mathbf{1}, w_{t}\right) \leq \frac{1}{2} e^{2 \alpha t}\left\|w_{t}\right\|^{2}+4 e^{2 \alpha t} w_{d}^{2}\|\nabla w\|^{2},
$$

and using Gagliardo-Nirenberg inequality and Lemma 3.1, by

$$
\begin{aligned}
2 e^{2 \alpha t} B\left(w ; w, w_{t}\right) \leq & C e^{2 \alpha t}\|w\|_{L^{4}}\|\nabla w\|_{L^{4}}\left\|w_{t}\right\| \\
\leq & C e^{2 \alpha t}\left(\|w\|^{\frac{1}{2}}\|\nabla w\|^{\frac{1}{2}}+\|w\|\right)\left(\|w\|^{\frac{1}{4}}\|\Delta w\|^{\frac{3}{4}}+\|w\|\right)\left\|w_{t}\right\| \\
\leq & C e^{2 \alpha t}\left(\|w\|^{\frac{3}{4}}\|\nabla w\|^{\frac{1}{2}}\|\Delta w\|^{\frac{3}{4}}\left\|w_{t}\right\|+\|w\|^{\frac{5}{4}}\|\Delta w\|^{\frac{3}{4}}\left\|w_{t}\right\|\right. \\
& \left.\quad+\|w\|^{\frac{3}{2}}\|\nabla w\|^{\frac{1}{2}}\left\|w_{t}\right\|+\|w\|^{2}\left\|w_{t}\right\|\right) \\
\leq & \frac{1}{2} e^{2 \alpha t}\left\|w_{t}\right\|^{2}+C e^{2 \alpha t}\|w\|^{2}\|\nabla w\|^{4}+C e^{2 \alpha t}\|\Delta w\|^{2}+C e^{2 \alpha t}\|w\|^{2} \\
& +C e^{2 \alpha t}\|w\|^{2}\|\nabla w\|^{2} .
\end{aligned}
$$

Hence, rewriting the boundary integral term in (3.11) as in previous Lemma 3.2, we arrive from (3.11) at

$$
\begin{aligned}
\frac{d}{d t}\left(e ^ { 2 \alpha t } \left(v\|\nabla w\|^{2}\right.\right. & \left.\left.+2\left(c_{0}+w_{d}\right)\|w\|_{L^{2}(\partial \Omega)}^{2}+\frac{1}{9 c_{0}}\|w\|_{L^{4}(\partial \Omega)}^{4}\right)\right)+\left\|e^{\alpha t} w_{t}\right\|^{2} \\
& \leq C e^{2 \alpha t}\left(\|w\|_{L^{2}(\partial \Omega)}^{2}+\|w\|_{L^{4}(\partial \Omega)}^{4}+\|\nabla w\|^{2}+\|\Delta w\|^{2}+\|w\|^{2}\|\nabla w\|^{2}\right. \\
& \left.+\|w\|^{2}\|\nabla w\|^{4}+\|w\|^{2}\right) .
\end{aligned}
$$

Apply Lemmas 3.1 and 3.2, and the Grönwall's inequality to the above inequality to complete the rest of the proof.

Lemma 3.4 Let $w_{0} \in H^{2}(\Omega)$. Then there exists a positive constant $C=C\left(\left\|w_{0}\right\|_{2}\right)$ such that

$$
\begin{gathered}
\left\|w_{t}(t)\right\|^{2}+\|\Delta w(t)\|^{2}+v e^{-2 \alpha t} \int_{0}^{t} e^{2 \alpha s}\left\|\nabla w_{t}(s)\right\|^{2} d s+2 e^{-2 \alpha t} \int_{0}^{t} e^{2 \alpha s}\left(2\left(c_{0}+w_{d}\right)\left\|w_{t}(s)\right\|_{L^{2}(\partial \Omega)}^{2}\right. \\
\left.+\frac{2}{3 c_{0}}\left\|w(s) w_{t}(s)\right\|_{L^{2}(\partial \Omega)}^{2}\right) d s \leq C e^{C} e^{-2 \alpha t} .
\end{gathered}
$$

Proof Differentiate (2.6) with respect to $t$ and then take the inner product with $e^{2 \alpha t} w_{t}$ to obtain

$$
\begin{aligned}
& \frac{d}{d t}\left(\left\|e^{\alpha t} w_{t}\right\|^{2}\right)-2 \alpha\left\|e^{\alpha t} w_{t}\right\|^{2}+2 v\left\|e^{\alpha t} \nabla w_{t}\right\|^{2}+2 \int_{\partial \Omega}\left(2\left(c_{0}+w_{d}\right) w_{t}^{2}+\frac{2}{3 c_{0}} w^{2} w_{t}^{2}\right) e^{2 \alpha t} d \Gamma \\
& =-2 e^{2 \alpha t}\left(B\left(w_{t} ; w, w_{t}\right)+B\left(w ; w_{t}, w_{t}\right)\right)-2 w_{d} e^{2 \alpha t}\left(\nabla w_{t} \cdot \mathbf{1}, w_{t}\right) .
\end{aligned}
$$


The right hand side terms in (3.12) are bounded by

$$
\begin{aligned}
-2 e^{2 \alpha t}( & \left.\left(\nabla w_{t} \cdot \mathbf{1}, w_{t}\right)+B\left(w_{t} ; w, w_{t}\right)+B\left(w ; w_{t}, w_{t}\right)\right) \\
\leq & 2 w_{d} e^{2 \alpha t}\left\|\nabla w_{t}\right\|\left\|w_{t}\right\|+C e^{2 \alpha t}\left\|w_{t}\right\|_{L^{4}}\|\nabla w\|\left\|w_{t}\right\|_{L^{4}}+C e^{2 \alpha t}\|w\|_{L^{4}}\left\|\nabla w_{t}\right\|\left\|w_{t}\right\|_{L^{4}} \\
\leq & C e^{2 \alpha t}\left\|\nabla w_{t}\right\|\left\|w_{t}\right\|+C e^{2 \alpha t}\left(\left\|w_{t}\right\|^{\frac{1}{2}}\left\|\nabla w_{t}\right\|^{\frac{1}{2}}+\left\|w_{t}\right\|\right)^{2}\|\nabla w\| \\
& \quad+C\left(\|w\|^{\frac{1}{2}}\|\nabla w\|^{\frac{1}{2}}+\|w\|\right)\left(\left\|w_{t}\right\|^{\frac{1}{2}}\left\|\nabla w_{t}\right\|^{\frac{1}{2}}+\left\|w_{t}\right\|\right)\left\|\nabla w_{t}\right\| \\
\leq & v\left\|e^{\alpha t} \nabla w_{t}\right\|^{2}+C e^{2 \alpha t}\left(\left\|w_{t}\right\|^{2}+\left\|w_{t}\right\|^{2}\|\nabla w\|^{2}+\|w\|^{2}\|\nabla w\|^{2}\left\|w_{t}\right\|^{2}+\|w\|^{2}\|\nabla w\|^{2}\right. \\
& \left.+\left\|w_{t}\right\|^{2}\|w\|^{2}+\left\|w_{t}\right\|^{2}\|w\|^{4}+\|w\|^{2}\right) .
\end{aligned}
$$

Hence, from (3.12), we arrive at

$$
\begin{aligned}
\frac{d}{d t}\left(\left\|e^{\alpha t} w_{t}\right\|^{2}\right)+v & \left\|e^{\alpha t} \nabla w_{t}\right\|^{2}+2 e^{2 \alpha t}\left(2\left(c_{0}+w_{d}\right)\left\|w_{t}\right\|_{L^{2}(\partial \Omega)}^{2}+\frac{2}{3 c_{0}}\left\|w w_{t}\right\|_{L^{2}(\partial \Omega)}^{2}\right) \\
\leq & C e^{2 \alpha t}\left(\left\|w_{t}\right\|^{2}+\left\|w_{t}\right\|^{2}\|\nabla w\|^{2}+\|w\|^{2}\|\nabla w\|^{2}\left\|w_{t}\right\|^{2}+\|w\|^{2}\|\nabla w\|^{2}\right. \\
& \left.+\left\|w_{t}\right\|^{2}\|w\|^{2}+\left\|w_{t}\right\|^{2}\|w\|^{4}+\|w\|^{2}\right) .
\end{aligned}
$$

To calculate $\left\|w_{t}(0)\right\|$, take the inner product between (2.6) and $w_{t}$; and use compatibility condition to obtain

$$
\left\|w_{t}(0)\right\|^{2} \leq C\left(\left\|\nabla w_{0}\right\|^{2}+\left\|\Delta w_{0}\right\|^{2}+\left\|w_{0}\right\|^{2}\left\|\nabla w_{0}\right\|^{4}\right) .
$$

Integrate the inequality (3.13) from 0 to $t$ and then use Lemmas 3.1-3.3 to complete the proof of $\left\|w_{t}(t)\right\|$. From this the estimate of $\|\Delta w(t)\|$ follows. Altogether it completes the rest of the proof.

Lemma 3.5 Let $w_{0} \in H^{3}(\Omega)$. Then there exists a positive constant $C=C\left(\left\|w_{0}\right\|_{3}\right)$ such that

$$
\begin{aligned}
\left\|\nabla w_{t}(t)\right\|^{2}+\left(2\left(c_{0}+w_{d}\right)\left\|w_{t}(t)\right\|_{L^{2}(\partial \Omega)}^{2}\right. & \left.+\frac{2}{3 c_{0}}\left\|w(t) w_{t}(t)\right\|_{L^{2}(\partial \Omega)}^{2}\right)+v e^{-2 \alpha t} \int_{0}^{t} e^{2 \alpha s}\left\|\Delta w_{t}(s)\right\|^{2} d s \\
& \leq C e^{C\left(\left\|w_{0}\right\|_{2}\right)} e^{-2 \alpha t} .
\end{aligned}
$$

Proof Differentiate (2.6) with respect to $t$ and then take inner product with $-e^{2 \alpha t} \Delta w_{t}$ to obtain

$$
\begin{aligned}
\frac{d}{d t}\left\|e^{\alpha t} \nabla w_{t}\right\|^{2}- & 2 \alpha\left\|e^{\alpha t} \nabla w_{t}\right\|^{2}+2 v\left\|e^{\alpha t} \Delta w_{t}\right\|^{2}+\frac{d}{d t} \int_{\partial \Omega} e^{2 \alpha t}\left(2\left(c_{0}+w_{d}\right) w_{t}^{2}+\frac{2}{3 c_{0}} w^{2} w_{t}^{2}\right) d \Gamma \\
\leq & 2 e^{2 \alpha t} w_{d}\left(\nabla w_{t} \cdot \mathbf{1}, \Delta w_{t}\right)+2 e^{2 \alpha t} B\left(w_{t}, w, \Delta w_{t}\right)+2 e^{2 \alpha t} B\left(w ; w_{t}, \Delta w_{t}\right) \\
& +C \int_{\partial \Omega} e^{2 \alpha t}\left(w_{t}^{2}+w w_{t}^{3}+w^{2} w_{t}^{2}\right) d \Gamma
\end{aligned}
$$

The first three terms on the right hand side of (3.14) are bounded by

$$
2 e^{2 \alpha t} w_{d}\left(\nabla w_{t} \cdot \mathbf{1}, \Delta w_{t}\right) \leq \frac{v}{3}\left\|e^{\alpha t} \Delta w_{t}\right\|^{2}+C e^{2 \alpha t}\left\|\nabla w_{t}\right\|^{2},
$$


and using Gagliardo-Nirenberg inequality and Lemma 3.1, by

$$
\begin{aligned}
2 e^{2 \alpha t}( & \left.B\left(w_{t} ; w, \Delta w_{t}\right)+B\left(w ; w_{t}, \Delta w_{t}\right)\right) \\
\leq & C e^{2 \alpha t}\left(\left\|w_{t}\right\|_{L^{4}}\|\nabla w\|_{L^{4}}\left\|\Delta w_{t}\right\|+\|w\|_{L^{4}}\left\|w_{t}\right\|_{L^{4}}\left\|\Delta w_{t}\right\|\right) \\
\leq & \frac{2 v}{3}\left\|e^{\alpha t} \Delta w_{t}\right\|^{2}+C e^{2 \alpha t}\left\|w_{t}\right\|^{2}\left(\|w\|_{2}^{2}+\|w\|^{2}\|\nabla w\|^{4}\right) \\
& +C e^{2 \alpha t}\left\|\nabla w_{t}\right\|^{2}\left(\|w\|^{2}+\|\Delta w\|^{2}\right) .
\end{aligned}
$$

The boundary terms on the right hand side of (3.14) are bounded by

$$
C \int_{\partial \Omega} e^{2 \alpha t}\left(w_{t}^{2}+w w_{t}^{3}+w^{2} w_{t}^{2}\right) d \Gamma \leq C \int_{\partial \Omega} e^{2 \alpha t}\left(w_{t}^{2}+w^{2} w_{t}^{2}\right) d \Gamma+C e^{2 \alpha t}\left\|w_{t}\right\|_{L^{4}(\partial \Omega)}^{4} .
$$

Therefore, from (3.14), we arrive at

$$
\begin{aligned}
\frac{d}{d t}\left(\left\|e^{\alpha t} \nabla w_{t}\right\|^{2}\right. & \left.+2\left(c_{0}+w_{d}\right)\left\|e^{\alpha t} w_{t}\right\|_{L^{2}(\partial \Omega)}^{2}+\frac{2}{3 c_{0}} \int_{\partial \Omega} e^{2 \alpha t} w^{2} w_{t}^{2} d \Gamma\right)+v\left\|e^{\alpha t} \Delta w_{t}\right\|^{2} \\
& \leq C e^{2 \alpha t}\left\|w_{t}\right\|^{2}\left(\|w\|_{2}^{2}+\|w\|^{2}\|\nabla w\|^{4}\right)+C e^{2 \alpha t}\left\|\nabla w_{t}\right\|^{2}\left(1+\|w\|^{2}+\|\Delta w\|^{2}\right) \\
& +\int_{\partial \Omega} e^{2 \alpha t}\left(w_{t}^{2}+w^{2} w_{t}^{2}\right) d s+C e^{2 \alpha t}\left(\left\|w_{t}\right\|^{4}+\left\|\nabla w_{t}\right\|^{4}\right) .
\end{aligned}
$$

Integrate the above inequality from 0 to $t$ and then apply the Grönwall's inequality along with Lemmas 3.1-3.4 to obtain

$$
\begin{gathered}
\left(\left\|e^{\alpha t} \nabla w_{t}(t)\right\|^{2}+2\left(c_{0}+w_{d}\right)\left\|e^{\alpha t} w_{t}(t)\right\|_{L^{2}(\partial \Omega)}^{2}+\frac{2}{3 c_{0}} \int_{\partial \Omega} e^{2 \alpha t} w(t)^{2} w_{t}(t)^{2} d \Gamma\right) \\
+v \int_{0}^{t}\left\|e^{\alpha s} \Delta w_{t}(s)\right\|^{2} d s \\
\leq C\left(\left\|\nabla w_{t}(0)\right\|^{2}+\left\|w_{t}(0)\right\|_{L^{2}(\partial \Omega)}+\left\|w(0) w_{t}(0)\right\|_{L^{2}(\partial \Omega)}^{2}\right) \\
\quad \exp \left(C \int_{0}^{t}\left(\|w(s)\|^{2}+\|\Delta w(s)\|^{2}+\left\|\nabla w_{t}(s)\right\|^{2}\right) d s\right) .
\end{gathered}
$$

Differentiate (2.6) with respect to $x_{1}$ and $x_{2}$ and applying compatibility condition to arrive at $\left\|\nabla w_{t}(0)\right\| \leq C\left\|w_{0}\right\|_{3}$. Also, by $(2.1),\left\|w_{t}(0)\right\|_{L^{2}(\partial \Omega)} \leq C\left\|w_{t}(0)\right\|_{1}^{2}$ and $\left\|w(0) w_{t}(0)\right\|_{L^{2}(\partial \Omega)}^{2} \leq C\|w(0)\|_{L^{4}(\partial \Omega)}\left\|w_{t}(0)\right\|_{1}^{2}$.

Again, use of Lemmas 3.1, 3.2 and 3.4 for the above inequality (3.15) completes the proof.

\section{Finite Element Method}

In this section, we discuss semidiscrete Galerkin approximation keeping the time variable continuous and show global stabilization result for the spatially discrete or semidiscrete solution. Further optimal error estimates ( optimality with respect to approximation property) for both state variable and feedback controller are derived. With respect to $H^{s}$ regularity of $w$, the optimal error estimate satisfy $\left\|w(t)-w_{h}(t)\right\|_{H^{m}(\Omega)} \leq C h^{\min (s, k+1)-m}\|w(t)\|_{H^{s}(\Omega)}$, where $m, s(0 \leq m<s)$ are integers and $k$ is the degree of polynomial used, Here $m=0$ or $1, k=1$ and $s=2$. 
For our semidiscrete analysis we assume $\Omega$ is a convex polygonal domain with boundary $\partial \Omega$, otherwise we can always get a polygonal domain $\Omega_{h} \subset \Omega$, where union of quasi-uniform triangulation determines $\Omega_{h}$ with boundary vertices on $\partial \Omega$. For more details see [31]. Given a regular triangulation $\mathcal{T}_{h}$ of $\bar{\Omega}$, let $h_{K}=\operatorname{diam}(K)$ for all $K \in \mathcal{T}_{h}$ and $h=\max _{K \in \mathcal{T}_{h}} h_{K}$.

Set

$$
V_{h}=\left\{v_{h} \in C^{0}(\bar{\Omega}):\left.v_{h}\right|_{K} \in \mathcal{P}_{1}(K) \quad \forall K \in \mathcal{T}_{h}\right\} .
$$

We shall assume further that the following inverse property hold for each $v_{h} \in V_{h}$ and $p \in[2, \infty]$, see, [9]

$$
\left\|v_{h}\right\|_{L^{p}(\Omega)} \leq C h^{2\left(\frac{1}{p}-\frac{1}{2}\right)}\left\|v_{h}\right\| .
$$

The semidiscrete approximation corresponding to the problem (2.14) is to seek $w_{h}(t)=$ $w_{h}(\cdot, t) \in V_{h}$ such that

$$
\begin{aligned}
& \left(w_{h t}, \chi\right)+v\left(\nabla w_{h}, \nabla \chi\right)+w_{d}\left(\nabla w_{h} \cdot \mathbf{1}, \chi\right)+B\left(w_{h} ; w_{h}, \chi\right) \\
& \quad+\int_{\partial \Omega}\left(2\left(c_{0}+w_{d}\right) w_{h}+\frac{2}{9 c_{0}} w_{h}^{3}\right) \chi d \Gamma=0, \quad \forall \chi \in V_{h}
\end{aligned}
$$

with $w_{h}(0)=P_{h} u_{0}-w_{d}=w_{0 h}$ (say), an approximation of $w_{0}$, where, $P_{h} u_{0}$ is the $H^{1}$ projection of $u_{0}$ onto $V_{h}$ such that

$$
\left\|u_{0}-u_{0 h}\right\|_{j} \leq C h^{2-j}\left\|u_{0}\right\|_{2} \quad j=0,1 .
$$

Since $V_{h}$ is finite dimensional, (4.2) leads to a system of nonlinear ODEs. Hence, an application of Picard's theorem ensures the existence of a unique solution locally, that is, there exists an interval $\left(0, t_{h}\right)$ such that $w_{h}$ exists for $t \in\left(0, t_{h}\right)$. Then, using the boundedness of the discrete solution from Lemma 4.1 below, the continuation arguments yields existence of a unique solution for all $t>0$.

In a similar fashion as in continuous case, the following stabilization result holds for the semidiscrete solution.

Lemma 4.1 Let $w_{0} \in L^{2}(\Omega)$.Then, there holds

$$
\begin{aligned}
& \left\|w_{h}(t)\right\|^{2}+\beta e^{-2 \alpha t} \int_{0}^{t} e^{2 \alpha s}\left(\left\|\nabla w_{h}(s)\right\|^{2}+\left\|w_{h}(s)\right\|_{L^{2}(\partial \Omega)}^{2}\right) d s+\frac{1}{3 c_{0}} e^{-2 \alpha t} \\
& \quad \times \int_{0}^{t} e^{2 \alpha s}\left\|w_{h}(s)\right\|_{L^{4}(\partial \Omega)}^{4} d s \\
& \leq e^{-2 \alpha t}\left\|w_{0 h}\right\|^{2} .
\end{aligned}
$$

\subsection{Error Estimates}

Define an auxiliary projection $\tilde{w}_{h} \in V_{h}$ of $w$ through the following form

$$
\left(\nabla\left(w-\tilde{w}_{h}\right), \nabla \chi\right)+\lambda\left(w-\tilde{w}_{h}, \chi\right)=0 \quad \forall \chi \in V_{h},
$$

where $\lambda \geq 1$ is some fixed positive number. For a given $w$, the existence of a unique $\tilde{w}_{h}$ follows by the Lax-Milgram Lemma. Let $\eta:=w-\tilde{w}_{h}$ be the error involved in the auxiliary 
projection. Then, the following error estimates hold:

$$
\begin{aligned}
\|\eta\|_{j} & \leq C h^{\min (2, m)-j}\|w\|_{m}, \text { and } \\
\left\|\eta_{t}\right\|_{j} & \leq C h^{\min (2, m)-j}\left\|w_{t}\right\|_{m}, \quad j=0,1 \text { and } m=1,2 .
\end{aligned}
$$

For a proof, we refer to Thomée [9,31]. Following Lemma 4.2 is needed to establish error estimates.

Lemma 4.2 Let $F \in H^{3 / 2+\epsilon}(\Omega)$, for some $\epsilon>0$, and $G \in H^{1 / 2}(\partial \Omega)$. Then $F G \in$ $H^{1 / 2}(\partial \Omega)$ and

$$
\|F G\|_{H^{1 / 2}(\partial \Omega)} \leq C\|F\|_{H^{3 / 2+\epsilon}(\Omega)}\|G\|_{H^{1 / 2}(\partial \Omega)} .
$$

Proof For a proof see [10].

In addition, for proving error estimates for state variable and feedback controllers, we need the following estimates of $\eta$ and $\eta_{t}$ at boundary.

Lemma 4.3 For smooth $\partial \Omega$, there holds

$$
\begin{aligned}
& \|\eta\|_{L^{2}(\partial \Omega)} \leq C h^{3 / 2}\|w\|_{2}, \quad\|\eta\|_{H^{-1 / 2}(\partial \Omega)} \leq C h^{2}\|w\|_{2}, \quad\left\|\eta_{t}\right\|_{H^{-1 / 2}(\partial \Omega)} \leq C h^{2}\left\|w_{t}\right\|_{2}, \\
& \|\eta\|_{L^{q}(\partial \Omega)} \leq C h\|w\|_{2}, \quad \text { and }\left\|\eta_{t}\right\|_{L^{q}(\partial \Omega)} \leq C h\left\|w_{t}\right\|_{2}, \quad 2 \leq q<\infty .
\end{aligned}
$$

Proof Consider an auxiliary function $\phi$ satisfying the following problem

$$
\begin{aligned}
-\Delta \phi+\lambda \phi & =0 \text { in } \Omega, \\
\frac{\partial \phi}{\partial v} & =\eta \text { on } \partial \Omega,
\end{aligned}
$$

with $\|\phi\|_{2} \leq C\|\eta\|_{H^{\frac{1}{2}(\partial \Omega)}}$. For a proof of this regularity result see [21].

Take the inner product between (4.6) and $\eta$ to obtain

$$
\begin{aligned}
\|\eta\|_{L^{2}(\partial \Omega)}^{2}=(\nabla \phi, \nabla \eta)+\lambda(\phi, \eta) & =\left(\nabla \phi-\nabla \tilde{\phi_{h}}, \nabla \eta\right)+\lambda\left(\phi-\tilde{\phi_{h}}, \eta\right) \\
& \leq c h^{2}\|\phi\|_{2}\|w\|_{2}+C h^{4}\|\phi\|_{2}\|w\|_{2} \leq C h^{2}\|w\|_{2}\|\eta\|_{H^{\frac{1}{2}(\partial \Omega)}} .
\end{aligned}
$$

Using the Trace inequality (2.2) for $s=1 / 2$, we arrive at

$$
\|\eta\|_{L^{2}(\partial \Omega)}^{2} \leq C h^{2}\|w\|_{2}^{2}\|\eta\|_{H^{1}(\Omega)} \leq C h^{3}\|w\|_{2}^{2} .
$$

Hence, $\|\eta\|_{L^{2}(\partial \Omega)} \leq C h^{\frac{3}{2}}\|w\|_{2}$.

The idea for showing estimate $\|\eta\|_{H^{-1 / 2}(\partial \Omega)}$ using variant of the Aubin-Nitsche technique can be found in [10]. For completeness, we provide a brief proof here. Let $\beta=\beta(t)$ be the solution of

$$
(\nabla \beta, \nabla \chi)+\lambda(\beta, \chi)=\langle\delta, \chi\rangle_{\partial \Omega},
$$

where $\delta \in H^{1 / 2}(\partial \Omega)$ is such that

$$
\|\delta\|_{H^{1 / 2}(\partial \Omega)}=\|\eta\|_{H^{-1 / 2}(\partial \Omega)}, \quad\langle\delta, \eta\rangle_{\partial \Omega}=\|\eta\|_{H^{-1 / 2}(\partial \Omega)}^{2},
$$

where the existence of $\delta$ follows from the Hahn-Banach theorem. Set $\chi=\eta$ and use (4.4) to obtain

$$
\begin{aligned}
\|\eta\|_{H^{-1 / 2}(\partial \Omega)}^{2} & =(\nabla \eta, \nabla \beta)+\lambda(\eta, \beta) \\
& =(\nabla \eta, \nabla(\beta-\phi))+\lambda(\eta, \beta-\phi) \leq C\|\eta\|_{1}\|\beta-\phi\|_{1} \quad \forall \phi \in V_{h} .
\end{aligned}
$$


Therefore using $\inf _{\chi \in V_{h}}\|v-\chi\|_{i} \leq C h^{2-i}\|v\|_{2}, \quad i=0,1$ and (4.6), it follows that

$$
\|\eta\|_{H^{-1 / 2}(\partial \Omega)}^{2} \leq C h\|\eta\|_{1}\|\beta\|_{2} \leq C h\|\eta\|_{1}\|\delta\|_{H^{1 / 2}(\partial \Omega)} \leq C h\|\eta\|_{1}\|\eta\|_{H^{-1 / 2}(\partial \Omega)} .
$$

Hence $\|\eta\|_{H^{-1 / 2}(\partial \Omega)} \leq C h^{2}\|w\|_{2}$.

Consider an auxiliary function $\phi$ satisfying the following problem

$$
\begin{aligned}
-\Delta \phi+\lambda \phi & =0 \text { in } \Omega, \\
\frac{\partial \phi}{\partial v} & =\eta_{t} \text { on } \partial \Omega,
\end{aligned}
$$

where $\|\phi\|_{2} \leq C\left\|\eta_{t}\right\|_{H^{\frac{1}{2}(\partial \Omega)}}$. For a proof of this regularity result see [21].

Similarly we can show that $\left\|\eta_{t}\right\|_{H^{-1 / 2}(\partial \Omega)} \leq C h^{2}\left\|w_{t}\right\|_{2}$.

Using (2.1), it follows for $q \in[2, \infty)$ that

$$
\|\eta\|_{L^{q}(\partial \Omega)} \leq C\|\eta\|_{1} \leq C h\|w\|_{2},
$$

and

$$
\left\|\eta_{t}\right\|_{L^{q}(\partial \Omega)} \leq C\left\|\eta_{t}\right\|_{1} \leq C h\left\|w_{t}\right\|_{2} .
$$

This completes the proof.

With $e:=w-w_{h}$, decompose $e:=\left(w-\tilde{w}_{h}\right)-\left(w_{h}-\tilde{w}_{h}\right)=: \eta-\theta$, where $\eta=w-\tilde{w}_{h}$ and $\theta=w_{h}-\tilde{w}_{h}$.

Since estimates of $\eta$ are known from (4.5) and Lemma 4.3, it is sufficient to estimate $\theta$. Subtracting the weak formulation (2.14) from (4.2) and a use of (4.4) yields

$$
\begin{aligned}
\left(\theta_{t}, \chi\right)+ & v(\nabla \theta, \nabla \chi)+\int_{\partial \Omega}\left(2\left(c_{0}+w_{d}\right) \theta+\frac{2}{9 c_{0}} \theta^{3}+\frac{2}{3 c_{0}} w_{h}^{2} \theta\right) \chi d \Gamma \\
= & \left(\eta_{t}-v \lambda \eta, \chi\right)+w_{d}(\nabla(\eta-\theta) \cdot \mathbf{1}, \chi)+\int_{\partial \Omega} 2\left(c_{0}+w_{d}\right) \eta \chi d \Gamma \\
& +\left((\eta-\theta) \nabla w \cdot \mathbf{1}+w_{h}(\nabla \eta-\nabla \theta) \cdot \mathbf{1}, \chi\right)+\frac{2}{9 c_{0}} \int_{\partial \Omega}\left(\eta^{3}+3 w \eta(w-\eta)+3 w_{h} \theta^{2}\right) \chi d \Gamma \\
= & \sum_{i=1}^{5} I_{i}(\chi) .
\end{aligned}
$$

Hence forward, we like to restrict the range of $\alpha$, that is,

$$
0 \leq \alpha \leq \frac{1}{C_{F}} \min \left\{3 v / 4,\left(\left(c_{0} / 2\right)+w_{d}\right)\right\}
$$

For such $\alpha$, the results of Sect. 3 are also valid.

In the following theorem, we estimate $\|\theta(t)\|$.

Theorem 4.1 Let $w_{0} \in H^{3}(\Omega)$. Then, there exists a positive constant $C=C\left(\left\|w_{0}\right\|_{3}\right)$ such that there holds

$$
\begin{gathered}
\|\theta(t)\|^{2}+\beta_{1} e^{-2 \alpha t} \int_{0}^{t} e^{2 \alpha s}\left(\|\nabla \theta(s)\|^{2}+\|\theta(s)\|_{L^{2}(\partial \Omega)}^{2}+\|\theta(s)\|_{L^{4}(\partial \Omega)}^{4}\right) d s \\
\leq C\left(\left\|w_{0}\right\|_{3}\right) \exp \left(\left\|w_{0}\right\|_{2}\right) h^{4} e^{-2 \alpha t}
\end{gathered}
$$

where $\beta_{1}=\min \left(\left(\frac{3 v}{2}-2 \alpha C_{F}\right),\left(\left(c_{0}+2 w_{d}\right)-2 \alpha C_{F}\right), \frac{1}{27 c_{0}}\right)>0$. 
Proof Set $\chi=\theta$ in (4.9) to obtain

$$
\frac{1}{2} \frac{d}{d t}\|\theta\|^{2}+v\|\nabla \theta\|^{2}+2\left(c_{0}+w_{d}\right)\|\theta\|_{L^{2}(\Omega)}^{2}+\frac{2}{9 c_{0}}\|\theta\|_{L^{4}(\Omega)}^{4}+\frac{2}{3 c_{0}} \int_{\partial \Omega} w_{h}^{2} \theta^{2} d \Gamma=\sum_{i=1}^{5} I_{i}(\theta) .
$$

The first term $I_{1}(\theta)$ on the right hand side of (4.10) is bounded by

$$
I_{1}(\theta)=\left(\eta_{t}-v \lambda \eta, \theta\right) \leq C\left(\|\eta\|^{2}+\left\|\eta_{t}\right\|^{2}\right)+\frac{\epsilon}{16}\|\theta\|^{2},
$$

where $\epsilon>0$ is a positive number which we choose later. For the second term $I_{2}(\theta)$ on the right hand side of (4.10), a use of the Cauchy-Schwarz inequality with Young's inequality and $\|\theta\|_{H^{1 / 2}(\partial \Omega)} \leq C\|\theta\|_{1}$ yields

$$
\begin{aligned}
I_{2}(\theta) & =w_{d}(\nabla(\eta-\theta) \cdot \mathbf{1}, \theta) \\
& =-w_{d}(\eta, \nabla \theta \cdot \mathbf{1})+w_{d} \sum_{i=1}^{2} \int_{\partial \Omega} \eta v_{i} \theta d \Gamma-\frac{w_{d}}{2} \sum_{i=1}^{2} \int_{\partial \Omega} \theta^{2} v_{i} d \Gamma \\
& \leq C\|\eta\|\|\nabla \theta\|+C\|\eta\|_{H^{-1 / 2}(\partial \Omega)}\|\theta\|_{H^{1 / 2}(\partial \Omega)}+w_{d}\|\theta\|_{L^{2}(\partial \Omega)}^{2} \\
& \leq \frac{v}{24}\|\nabla \theta\|^{2}+\frac{\epsilon}{16}\|\theta\|^{2}+C\|\eta\|^{2}+w_{d}\|\theta\|_{L^{2}(\partial \Omega)}^{2}+C\|\eta\|_{H^{-1 / 2}(\partial \Omega)}^{2} .
\end{aligned}
$$

The third term $I_{3}(\theta)$ on the right hand side is bounded by

$$
\begin{gathered}
2\left(c_{0}+w_{d}\right)\langle\eta, \theta\rangle_{(\partial \Omega)} \leq C\|\eta\|_{H^{-1 / 2}(\partial \Omega)}\|\theta\|_{H^{1 / 2}(\partial \Omega)} \leq C\|\eta\|_{H^{-1 / 2}(\partial \Omega)}(\|\nabla \theta\|+\|\theta\|) \\
\leq \frac{v}{24}\|\nabla \theta\|^{2}+\frac{\epsilon}{16}\|\theta\|^{2}+C\|\eta\|_{H^{-1 / 2}(\partial \Omega)}^{2} .
\end{gathered}
$$

For the fourth term $I_{4}(\theta)$, first we use the Gagliardo-Nirenberg inequality for $H^{1}$ function to bound the following sub-terms as

$$
\begin{aligned}
& ((\eta-\theta) \nabla w \cdot \mathbf{1}, \theta) \leq C\|\eta\|\|\nabla w\|_{L^{4}}\|\theta\|_{L^{4}}+C\|\theta\|\|\theta\|_{L^{4}}\|\nabla w\|_{L^{4}} \\
& \quad \leq \frac{v}{48}\|\nabla \theta\|^{2}+\frac{\epsilon}{16}\|\theta\|^{2}+C\|\theta\|^{2}\|w\|_{2}^{2}+C\|\eta\|^{2}\left(1+\|w\|^{2}+\|\Delta w\|^{2}\right)
\end{aligned}
$$

and apply $w_{h}=\theta+\tilde{w}_{h}$ with integration by parts and $\left\|\tilde{w}_{h}\right\|_{L^{\infty}} \leq C\|w\|_{2}$ to obtain a bound

$$
\begin{aligned}
-\left(w_{h} \nabla \theta \cdot \mathbf{1}, \theta\right) & =-(\theta \nabla \theta \cdot \mathbf{1}, \theta)-\left(\tilde{w}_{h} \nabla \theta \cdot \mathbf{1}, \theta\right)=-\frac{1}{3} \sum_{j=1}^{2} \int_{\partial \Omega} \theta^{3} n_{j} d \Gamma-\left(\tilde{w}_{h} \nabla \theta \cdot \mathbf{1}, \theta\right) \\
& \leq \frac{\sqrt{2}}{3} \int_{\partial \Omega}|\theta|^{3} d \Gamma+\left\|\tilde{w}_{h}\right\|_{L^{\infty}}\|\nabla \theta\|\|\theta\| \\
& \leq \frac{3 c_{0}}{2} \int_{\partial \Omega}|\theta|^{2} d \Gamma+\frac{1}{27 c_{0}} \int_{\partial \Omega}|\theta|^{4} d \Gamma+\frac{v}{48}\|\nabla \theta\|^{2}+C\|\theta\|^{2}\|w\|_{2}^{2} .
\end{aligned}
$$


Using the Sobolev inequality $\|\theta\|_{L^{4}} \leq C\|\theta\|_{1}$ and $\left\|\tilde{w}_{h} \theta\right\|_{H^{1 / 2}(\partial \Omega)} \leq C\|w\|_{2} \quad\|\theta\|_{1}$, the other sub-term in $I_{4}(\theta)$ can be bounded by

$$
\begin{aligned}
\left(w_{h} \nabla \eta \cdot \mathbf{1}, \theta\right)= & (\theta \nabla \eta \cdot \mathbf{1}, \theta)-\left(\tilde{w}_{h} \nabla \theta \cdot \mathbf{1}, \eta\right)-\left(\eta \nabla \tilde{w}_{h} \cdot \mathbf{1}, \theta\right)+\sum_{i=1}^{2} \int_{\partial \Omega} \tilde{w}_{h} \eta v_{i} \theta d \Gamma \\
\leq & \|\theta\|\|\nabla \eta\|_{L^{4}}\|\theta\|_{L^{4}}+\|\eta\|\|\nabla \theta\|\left\|\tilde{w}_{h}\right\|_{L^{\infty}}+\|\eta\|\left\|\nabla \tilde{w}_{h}\right\|_{L^{4}}\|\theta\|_{L^{4}} \\
& +\|\eta\|_{H^{-1 / 2}(\partial \Omega)}\left\|\tilde{w}_{h} \theta\right\|_{H^{1 / 2}(\partial \Omega)} \\
\leq & \frac{v}{24}\|\nabla \theta\|^{2}+\frac{\epsilon}{16}\|\theta\|^{2}+C\left(\|w\|_{2}^{2}\|\eta\|^{2}+\|\eta\|_{H^{-1 / 2}(\partial \Omega)}^{2}\right)+C\|\theta\|^{2}\left(\|w\|^{2}+\|\nabla \eta\|_{L^{4}}^{2}\right) .
\end{aligned}
$$

For $I_{5}(\theta)$, we note that

$$
\begin{aligned}
\frac{2}{9 c_{0}} \int_{\partial \Omega} \eta^{3} \theta d \Gamma & \leq C\|\eta\|_{L^{6}(\partial \Omega)}^{3}\|\theta\|_{L^{2}(\partial \Omega)} \leq C\|\eta\|_{L^{6}(\partial \Omega)}^{3}(\|\nabla \theta\|+\|\theta\|) \\
& \leq \frac{v}{48}\|\nabla \theta\|^{2}+\frac{\epsilon}{16}\|\theta\|^{2}+C\|\eta\|_{L^{6}(\partial \Omega)}^{6}, \\
\frac{2}{9 c_{0}} \int_{\partial \Omega} 3 w^{2} \eta \theta d \Gamma & \leq C\|\eta\|_{H^{-1 / 2}(\partial \Omega)}\left\|w^{2} \theta\right\|_{H^{1 / 2}(\partial \Omega)} \\
& \leq C\|\eta\|_{H^{-1 / 2}(\partial \Omega)}\|w\|_{2}\|w \theta\|_{H^{1 / 2}(\partial \Omega)} \\
& \leq C\|\eta\|_{H^{-1 / 2}(\partial \Omega)}\|w\|_{2}^{2}\|\theta\|_{1} \\
& \leq \frac{\epsilon}{16}\|\theta\|^{2}+\frac{v}{48}\|\nabla \theta\|^{2}+C\|\eta\|_{H^{-1 / 2}(\partial \Omega)}^{2}\|w\|_{2}^{4} \\
\frac{2}{9 c_{0}} \int_{\partial \Omega} 3 w \eta^{2} \theta d \Gamma & \leq \frac{\epsilon}{16}\|\theta\|^{2}+\frac{v}{24}\|\nabla \theta\|^{2}+C\|w\|_{L^{4}(\partial \Omega)}^{2}\|\eta\|_{L^{4}(\partial \Omega)}^{4}
\end{aligned}
$$

and

$$
\frac{2}{9 c_{0}} \int_{\partial \Omega} 3 w_{h} \theta^{3} d \Gamma \leq \frac{2}{3 c_{0}}\left(\int_{\partial \Omega} w_{h}^{2} \theta^{2} d \Gamma+\frac{1}{4} \int_{\partial \Omega} \theta^{4} d \Gamma\right) .
$$

Finally, using Lemmas 3.1-3.4, 4.1 and 4.3, we arrive from (4.10) at

$$
\begin{aligned}
\frac{d}{d t}\|\theta\|^{2}+ & \frac{3}{2} v\|\nabla \theta\|^{2}+\left(c_{0}+2 w_{d}\right)\|\theta\|_{L^{2}(\partial \Omega)}^{2}+\frac{1}{27 c_{0}}\|\theta\|_{L^{4}(\partial \Omega)}^{4} \\
\leq & \epsilon \theta\left\|^{2}+C\right\| \eta\left\|^{2}\left(1+\|w\|^{2}+\|\Delta w\|^{2}\right)+C\right\| \eta\left\|_{L^{6}(\partial \Omega)}^{6}+C\right\| \theta\left\|^{2}\left(1+h^{2}\right)\right\| w \|_{2}^{2} \\
& \quad+C\left(\|\eta\|_{L^{4}(\partial \Omega)}^{4}+\|\eta\|_{H^{-1 / 2}(\partial \Omega)}^{2}\right)\left(1+\|w\|_{2}^{2}+\|w\|_{L^{4}(\partial \Omega)}^{4}\right) .
\end{aligned}
$$

Multiply (4.11) by $e^{2 \alpha t}$ and use Friedrichs's inequality

$$
-2 \alpha e^{2 \alpha t}\|\theta\|^{2} \geq-2 \alpha C_{F} e^{2 \alpha t}\|\nabla \theta\|^{2}-2 \alpha C_{F} e^{2 \alpha t}\|\theta\|_{L^{2}(\partial \Omega)}^{2} .
$$

Then a use of Lemmas 3.1, 3.2, and 4.3in (4.11) yields

$$
\begin{aligned}
\frac{d}{d t}( & \left.\left\|e^{\alpha t} \theta\right\|^{2}\right)+e^{2 \alpha t}\left(\left(\frac{3 v}{2}-2 \alpha C_{F}\right)\|\nabla \theta\|^{2}+\left(\left(c_{0}+2 w_{d}\right)-2 \alpha C_{F}\right)\|\theta\|_{L^{2}(\partial \Omega)}^{2}+\frac{1}{27 c_{0}}\|\theta\|_{L^{4}(\partial \Omega)}^{4}\right) \\
\leq & C e^{2 \alpha t}\left(\|\eta\|^{2}+\|\eta\|^{2}+\|\eta\|_{H^{-1 / 2}(\partial \Omega)}^{2}+C\|\eta\|_{L^{4}(\partial \Omega)}^{4}\right. \\
& \left.+\|\eta\|_{L^{6}(\partial \Omega)}^{6}\right)+C e^{2 \alpha t}\|\theta\|^{2}\left(\left(1+h^{2}\right)\|w\|_{2}^{2}\right) \\
& +\epsilon C_{F} e^{2 \alpha t}\left(\|\nabla \theta\|^{2}+\|\theta\|_{L^{2}(\partial \Omega)}^{2}\right) .
\end{aligned}
$$


Integrate the above inequality from 0 to $t$ and choose $\epsilon=\frac{\beta_{1}}{2 C_{F}}$. Then use the Grönwall's inequality to obtain

$$
\begin{aligned}
& \left\|e^{\alpha t} \theta(t)\right\|^{2}+\beta_{1} \int_{0}^{t} e^{2 \alpha s}\left(\|\nabla \theta(s)\|^{2}+\|\theta(s)\|_{L^{2}(\partial \Omega)}^{2}+\|\theta(s)\|_{L^{4}(\partial \Omega)}^{4}\right) d s \\
& \leq C h^{4}\left(\int_{0}^{t}\left(\|w(s)\|_{2}^{2}+\left\|w_{t}(s)\right\|_{2}^{2}\right) d s\right) \exp \left(\int_{0}^{t}\left(\left(1+h^{2}\right)\|w(s)\|_{2}^{2}\right) d s\right) .
\end{aligned}
$$

A use of Lemmas 3.1-3.5, and 4.1to the above inequality with a multiplication of $e^{-2 \alpha t}$ completes the proof.

Remark 4.1 As a consequence of Theorem 4.1, we use inverse property (4.1) to arrive for $p \in[2, \infty]$ at

$$
\begin{aligned}
\left\|w_{h}(t)\right\|_{L^{p}(\Omega)} & \leq\left\|\tilde{w}_{h}(t)\right\|_{L^{p}(\Omega)}+\|\theta(t)\|_{L^{p}(\Omega)} \\
& \leq C\|w(t)\|_{2}+C h^{2\left(\frac{1}{p}-\frac{1}{2}\right)}\|\theta(t)\| \leq C e^{-\alpha t} \leq C .
\end{aligned}
$$

Theorem 4.2 Let $w_{0} \in H^{3}(\Omega)$. Then, there is a positive constant $C$ independent of $h$ such that

$$
\begin{gathered}
v\|\nabla \theta(t)\|^{2}+2\left(c_{0}+w_{d}\right)\|\theta(t)\|_{L^{2}(\partial \Omega)}^{2}+\frac{1}{9 c_{0}}\|\theta(t)\|_{L^{4}(\partial \Omega)}^{4}+e^{-2 \alpha t} \int_{0}^{t} e^{2 \alpha s}\left\|\theta_{t}(s)\right\|^{2} d s \\
\leq C\left(\left\|w_{0}\right\|_{3}\right) \exp \left(C\left\|w_{0}\right\|_{2}\right) h^{4} e^{-2 \alpha t} .
\end{gathered}
$$

Proof Set $\chi=\theta_{t}$ in (4.9) to obtain

$$
\begin{gathered}
\left\|\theta_{t}\right\|^{2}+\frac{1}{2} \frac{d}{d t}\left(v\|\nabla \theta\|^{2}+2\left(c_{0}+w_{d}\right)\|\theta\|_{L^{2}(\partial \Omega)}^{2}+\frac{1}{9 c_{0}}\|\theta\|_{L^{4}(\partial \Omega)}^{4}+\frac{2}{3 c_{0}}\left\|w_{h} \theta\right\|_{L^{2}(\partial \Omega)}^{2}\right) \\
=\sum_{i=1}^{4} I_{i}\left(\theta_{t}\right)+\left(I_{5}\left(\theta_{t}\right)+\frac{2}{3 c_{0}}\left\langle w_{h} w_{h t} \theta, \theta\right\rangle_{\partial \Omega}\right) .
\end{gathered}
$$

The first term $I_{1}\left(\theta_{t}\right)$ on the right hand side of (4.9) is bounded by

$$
I_{1}\left(\theta_{t}\right)=\left(\eta_{t}-\lambda \nu \eta, \theta_{t}\right) \leq \frac{1}{6}\left\|\theta_{t}\right\|^{2}+C\left(\|\eta\|^{2}+\left\|\eta_{t}\right\|^{2}\right) .
$$

The second term $I_{2}\left(\theta_{t}\right)$ on the right hand side of (4.9) can be rewritten as

$$
\begin{aligned}
I_{2}\left(\theta_{t}\right)= & w_{d}\left((\nabla \eta-\nabla \theta) \cdot \mathbf{1}, \theta_{t}\right) \\
= & -w_{d} \frac{d}{d t}(\eta, \nabla \theta \cdot \mathbf{1})+w_{d}\left(\eta_{t}, \nabla \theta \cdot \mathbf{1}\right)+w_{d} \frac{d}{d t}\left(\sum_{i=1}^{2} \int_{\partial \Omega} \eta v_{i} \theta d \Gamma\right) \\
& -w_{d} \sum_{i=1}^{2} \int_{\partial \Omega} \eta_{t} v_{i} \theta d \Gamma-w_{d}\left(\nabla \theta \cdot \mathbf{1}, \theta_{t}\right),
\end{aligned}
$$


and hence, we get

$$
\begin{aligned}
I_{2}\left(\theta_{t}\right)= & w_{d}\left((\nabla \eta-\nabla \theta) \cdot \mathbf{1}, \theta_{t}\right) \\
\leq & -w_{d} \frac{d}{d t}(\eta, \nabla \theta \cdot \mathbf{1})+w_{d} \frac{d}{d t}\left(\sum_{i=1}^{2} \int_{\partial \Omega} \eta v_{i} \theta d \Gamma\right) \\
& +\frac{1}{12}\left\|\theta_{t}\right\|^{2}+C\left(\|\theta\|^{2}+\|\nabla \theta\|^{2}\right)+C\left(\left\|\eta_{t}\right\|^{2}+\left\|\eta_{t}\right\|_{H^{-1 / 2}(\partial \Omega)}^{2}\right) .
\end{aligned}
$$

The third term $I_{3}\left(\theta_{t}\right)$ on the right hand side of (4.9) is bounded by

$$
\begin{aligned}
I_{3}\left(\theta_{t}\right) & =\left\langle 2\left(c_{0}+w_{d}\right) \eta, \theta_{t}\right\rangle_{\partial \Omega}=\frac{d}{d t}\left\langle 2\left(c_{0}+w_{d}\right) \eta, \theta\right\rangle-2\left(c_{0}+w_{d}\right)\left\langle\eta_{t}, \theta\right\rangle \\
& \leq \frac{d}{d t}\left\langle 2\left(c_{0}+w_{d}\right) \eta, \theta\right\rangle+C\left(\|\theta\|^{2}+\|\nabla \theta\|^{2}\right)+C\left\|\eta_{t}\right\|_{H^{-1 / 2}(\partial \Omega)}^{2} .
\end{aligned}
$$

For the fourth term $I_{4}\left(\theta_{t}\right)$ on the right hand side of (4.9), first we rewrite the sub terms as

$$
\left(\eta \nabla w \cdot \mathbf{1}, \theta_{t}\right)=\frac{d}{d t}((\eta \nabla w \cdot \mathbf{1}, \theta))-\left(\eta_{t} \nabla w \cdot \mathbf{1}, \theta\right)-\left(\eta(\nabla w \cdot \mathbf{1})_{t}, \theta\right),
$$

and bound it using the Gagliardo-Nirenberg inequality for $H^{2}$ function

$$
\begin{gathered}
\left(\eta \nabla w \cdot \mathbf{1}, \theta_{t}\right) \leq \\
\frac{d}{d t}((\eta \nabla w \cdot \mathbf{1}, \theta))+C\left\|\eta_{t}\right\|^{2}\left(1+\|w\|_{2}^{2}\right)+C\|\theta\|^{2}\left(1+\|\Delta w\|^{2}+\left\|\Delta w_{t}\right\|^{2}\right) \\
+C\|\nabla \theta\|^{2}\left(\|w\|^{2}+\left\|w_{t}\right\|^{2}\right)+C\|\eta\|^{2}\left\|\Delta w_{t}\right\|^{2} .
\end{gathered}
$$

For the other sub-term of $I_{4}\left(\theta_{t}\right)$, apply $w_{h}=\theta+\tilde{w}_{h}$ with and then use integration by parts to obtain

$$
\begin{aligned}
\left(w_{h} \nabla \eta \cdot \mathbf{1}, \theta_{t}\right)= & \left(\theta \nabla \eta \cdot \mathbf{1}, \theta_{t}\right)-\frac{d}{d t}\left(\left(\eta \nabla \theta \cdot \mathbf{1}, \tilde{w}_{h}\right)+\left(\eta\left(\nabla \tilde{w}_{h} \cdot \mathbf{1}\right), \theta\right)-\sum_{i=1}^{2} \int_{\partial \Omega} \eta \tilde{w}_{h} v_{i} \theta d \Gamma\right) \\
& +\left(\left(\eta \tilde{w}_{h}\right)_{t}, \nabla \theta \cdot \mathbf{1}\right)+\left(\left(\eta \nabla \tilde{w}_{h} \cdot \mathbf{1}\right)_{t}, \theta\right)-\sum_{i=1}^{2} \int_{\partial \Omega}\left(\eta \tilde{w}_{h}\right)_{t} v_{i} \theta d \Gamma,
\end{aligned}
$$

and hence, from $\|\theta\|_{L^{4}(\Omega)} \leq C(\|\nabla \theta\|+\|\theta\|)$ and $\left\|\tilde{w}_{h}\right\|_{L^{\infty}} \leq C(1+h)\|w\|_{2}$, (see, Ciarlet $[9$, page 168]), it follows that

$$
\begin{aligned}
\left(w_{h} \nabla \eta \cdot \mathbf{1}, \theta_{t}\right) \leq & -\frac{d}{d t}\left(\left(\eta \nabla \theta \cdot \mathbf{1}, \tilde{w}_{h}\right)+\left(\eta\left(\nabla \tilde{w}_{h} \cdot \mathbf{1}\right), \theta\right)-\sum_{i=1}^{2} \int_{\partial \Omega} \eta \tilde{w}_{h} v_{i} \theta d \Gamma\right) \\
& +C\left\|\eta_{t}\right\|^{2}\left(1+\|w\|_{2}^{2}\right)+C\|\eta\|^{2}\left(1+\left\|w_{t}\right\|_{2}^{2}\right) \\
& +C\left(\|\theta\|^{2}+\|\nabla \theta\|^{2}\right)\left(\|\nabla \eta\|_{L^{4}}^{2}+\left\|\nabla \eta_{t}\right\|_{L^{4}}^{2}+\|w\|_{2}^{2}+\left\|w_{t}\right\|_{2}^{2}\right) \\
& +C\left(\|\eta\|_{H^{-1 / 2}(\partial \Omega)}^{2}+\left\|\eta_{t}\right\|_{H^{-1 / 2}(\partial \Omega)}^{2}\right)+\frac{1}{12}\left\|\theta_{t}\right\|^{2} .
\end{aligned}
$$


For the remaining other two sub-terms of $I_{4}\left(\theta_{t}\right)$, a use of $\|\theta(t)\|_{L^{\infty}} \leq C h e^{-\alpha t} \leq C$ now leads to

$$
\begin{aligned}
-\left(\theta(\nabla w \cdot \mathbf{1}), \theta_{t}\right) & -\left(w_{h}(\nabla \theta \cdot \mathbf{1}), \theta_{t}\right)=-\left(\theta(\nabla w \cdot \mathbf{1}), \theta_{t}\right)-\left(\left(\theta+\tilde{w}_{h}\right) \nabla \theta \cdot \mathbf{1}, \theta_{t}\right) \\
& \leq\left(\|\nabla w\|_{L^{4}}\|\theta\|_{L^{4}}+\left(\|\theta\|_{L^{\infty}}+\left\|\tilde{w}_{h}\right\|_{L^{\infty}}\right)\|\nabla \theta\|\right)\left\|\theta_{t}\right\| \\
& \leq \frac{1}{12}\left\|\theta_{t}\right\|^{2}+C\|\theta\|^{2}\|w\|_{2}^{2}+\|\nabla \theta\|^{2}\left(\|w\|_{2}^{2}+1\right) .
\end{aligned}
$$

For the last term on the right hand side of (4.9), the first sub-term is bounded by

$$
\begin{aligned}
\frac{2}{9 c_{0}} \int_{\partial \Omega} \eta^{3} \theta_{t} d \Gamma & =\frac{2}{9 c_{0}} \frac{d}{d t}\left(\int_{\partial \Omega} \eta^{3} \theta d \Gamma\right)-\frac{2}{3 c_{0}} \int_{\partial \Omega} \eta^{2} \eta_{t} \theta d \Gamma \\
& \leq \frac{2}{9 c_{0}} \frac{d}{d t}\left(\int_{\partial \Omega} \eta^{3} \theta d \Gamma\right)+C\left(\|\eta\|_{L^{4}(\partial \Omega)}^{4}+\left\|\eta_{t}\right\|_{L^{4}(\partial \Omega)}^{2}\|\theta\|_{1}^{2}\right) .
\end{aligned}
$$

Similarly, the other sub-terms are bounded by

$$
\begin{aligned}
\frac{2}{9 c_{0}} \int_{\partial \Omega} 3 w^{2} \eta \theta_{t} d \Gamma \leq & \frac{2}{3 c_{0}} \frac{d}{d t}\left(\int_{\partial \Omega} w^{2} \eta \theta d \Gamma\right)+C\left\|\eta_{t}\right\|_{H^{-1 / 2}(\partial \Omega)}\|w\|_{2}^{2}\|\theta\|_{1} \\
& +C\|\eta\|_{H^{-1 / 2}(\partial \Omega)}\|w\|_{2}\left\|w_{t}\right\|_{2}\|\theta\|_{1} \\
\leq & \frac{2}{3 c_{0}} \frac{d}{d t}\left(\int_{\partial \Omega} w^{2} \eta \theta d \Gamma\right)+C\|w\|_{2}^{2}\left(\|\theta\|^{2}+\|\nabla \theta\|^{2}\right) \\
& +C\left\|\eta_{t}\right\|_{H^{-1 / 2}(\partial \Omega)}^{2}+C\|\eta\|_{H^{-1 / 2}(\partial \Omega)}^{2}\left\|w_{t}\right\|_{2}^{2}, \\
-\frac{2}{9 c_{0}} 3 \int_{\partial \Omega} w \eta^{2} \theta_{t} d \Gamma \leq & -\frac{2}{3 c_{0}} \frac{d}{d t}\left(\int_{\partial \Omega} w \eta^{2} \theta d \Gamma\right)+C\|\eta\|_{L^{4}(\partial \Omega)}^{4} \\
& +C\|\eta\|_{L^{4}(\partial \Omega)}^{2}\left\|\eta_{t}\right\|_{L^{4}(\partial \Omega)}^{2}+C\|\theta\|_{1}^{2}\left(\|w\|_{1}^{2}+\left\|w_{t}\right\|_{1}^{2}\right) .
\end{aligned}
$$

Trace inequality for fractional Sobolev norm yields

$$
\left\|v_{h}\right\|_{L^{\infty}(\partial K)} \leq C\left(\left\|v_{h}\right\|_{L^{\infty}(K)}+h_{K}^{\delta}\left\|v_{h}\right\|_{W^{\delta, \infty}(K)}\right) .
$$

Using inverse inequality $\left\|v_{h}\right\|_{W^{\delta, \infty}(K)} \leq C h^{-\delta}\left\|v_{h}\right\|_{L^{\infty}(K)}$, we obtain $\left\|v_{h}\right\|_{L^{\infty}(\partial K)} \leq$ $C\left\|v_{h}\right\|_{L^{\infty}(K)}$.

A use of $\|\theta(t)\|_{L^{\infty}} \leq C h e^{-\alpha t}$ with $w_{h t}=\theta_{t}+\tilde{w}_{h t},\left\|v_{h}\right\|_{L^{\infty}(\partial K \cap \partial \Omega)} \leq\left\|v_{h}\right\|_{L^{\infty}(\Omega)}$, and inverse inequality $\|\theta\|_{1} \leq C h^{-1}\|\theta\|$ yields

$$
\begin{aligned}
\frac{2}{3 c_{0}} \int_{\partial \Omega}\left(w_{h} \theta^{2} \theta_{t}\right. & \left.+w_{h} w_{h t} \theta^{2}\right) d \Gamma=\frac{2}{3 c_{0}} \int_{\partial \Omega}\left(2 w_{h} \theta^{2} \theta_{t}+w_{h} \tilde{w}_{h t} \theta^{2}\right) d \Gamma \\
& \leq C\left\|w_{h}\right\|_{L^{\infty}}\left(\|\theta\|_{L^{\infty}}\|\theta\|_{L^{2}(\partial \Omega)}\left\|\theta_{t}\right\|_{L^{2}(\partial \Omega)}+\left(\left\|w_{h}\right\|_{L^{\infty}}^{2}+\left\|\tilde{w}_{h t}\right\|_{L^{\infty}}^{2}\right)\|\theta\|_{L^{2}(\partial \Omega)}^{2}\right) \\
& \leq C\left(h\|w\|_{2}\|\theta\|_{L^{2}(\partial \Omega)}\left\|\theta_{t}\right\|_{1}+\left(\left\|w_{t}\right\|_{2}^{2}+\|w\|_{2}^{2}\right)\|\theta\|_{L^{2}(\partial \Omega)}^{2}\right) \\
& \leq C\left(\left\|w_{t}\right\|_{2}^{2}+\|w\|_{2}^{2}\right)\|\theta\|_{L^{2}(\partial \Omega)}^{2}+\frac{1}{12}\left\|\theta_{t}\right\|^{2} .
\end{aligned}
$$

Substitute all the above estimates in (4.13), apply kickback argument and then multiply the resulting ones by $e^{2 \alpha t}$ with using Lemmas 3.1, 3.4, 4.1 and 4.2 to obtain after setting

$$
\|\mid \theta(t)\|:=\left(v\|\nabla \theta\|^{2}+2\left(c_{0}+w_{d}\right)\|\theta\|_{L^{2}(\partial \Omega)}^{2}+\frac{1}{9 c_{0}}\|\theta\|_{L^{4}(\partial \Omega)}^{4}+\frac{2}{3 c_{0}}\left\|w_{h} \theta\right\|_{L^{2}(\partial \Omega)}^{2}\right)^{1 / 2}
$$


and

$$
\begin{aligned}
F\left(\eta, w_{h}\right)(\theta):= & \left(\left(\eta\left(\nabla w_{h} \cdot \mathbf{1}\right), \theta\right)+\left(\eta(\nabla \theta \cdot \mathbf{1}), w_{h}\right)-(\eta(\nabla w \cdot \mathbf{1}), \theta)\right. \\
& \left.-\sum_{i=1}^{2} \int_{\partial \Omega} \eta w_{h} v_{i} \theta d \Gamma-\left(2 c_{0}+2 w_{d}\right)<\eta, \theta>_{\partial \Omega}\right)
\end{aligned}
$$

as

$$
\begin{aligned}
\frac{d}{d t}\left(e^{2 \alpha t}\left\||\theta(t) \||^{2}\right)\right. & +e^{2 \alpha t}\left\|\theta_{t}\right\|^{2} \leq 2 \alpha e^{2 \alpha t}\|\mid \theta(t)\|^{2}-\frac{d}{d t}\left(e^{2 \alpha t} F\left(\eta, w_{h}\right)(\theta)\right)+2 \alpha e^{2 \alpha t} F\left(\eta, w_{h}\right)(\theta) \\
& +C e^{2 \alpha t}\left(\|\eta\|^{2}+\left\|\eta_{t}\right\|^{2}+\|\eta\|_{L^{4}(\partial \Omega)}^{2}+\|\eta\|_{H^{-1 / 2}(\partial \Omega)}^{2}+\left\|\eta_{t}\right\|_{H^{-1 / 2}(\partial \Omega)}^{2}\right) \\
& +C e^{2 \alpha t}\|\theta\|^{2}\left(1+\|w\|_{2}^{2}+\left\|w_{t}\right\|_{2}^{2}+\left\|\eta_{t}\right\|_{L^{4}(\partial \Omega)}^{2}\right)+C e^{2 \alpha t}\|\theta\|_{L^{4}(\partial \Omega)}^{4}\left\|w_{t}\right\|_{2}^{2} \\
& +C e^{2 \alpha t}\|\nabla \theta\|^{2}\left(\|w\|_{2}^{2}+\left\|w_{t}\right\|_{2}^{2}+\left\|\eta_{t}\right\|_{L^{4}(\partial \Omega)}^{2}\right)+C e^{2 \alpha t}\|\nabla \theta\|^{2} .
\end{aligned}
$$

Integrate the above inequality from 0 to $t$. Note that using the bound $\left\|w_{h}\right\|_{L^{\infty}} \leq C\|w\|_{2}$, we obtain

$$
2 \alpha \int_{0}^{t} e^{2 \alpha s}\left\||\theta(s) \||^{2} d s \leq C \int_{0}^{t} e^{2 \alpha s}\left(\|\nabla \theta\|^{2}+\|\theta\|_{L^{2}(\partial \Omega)}^{2}+\|\theta\|_{L^{4}(\partial \Omega)}^{4}\right) d s\right.
$$

and using the splitting $w_{h}=\theta+\tilde{w}_{h}$ for first term of $F$, we bound $F$ as

$$
\begin{aligned}
F\left(\eta, w_{h}\right)(\theta) & \leq C\left(\|\eta\|_{L^{4}(\Omega)}\|\theta\|+\|\eta\|\|w\|_{2}+\|w\|_{2}\|\eta\|_{H^{-1 / 2}(\partial \Omega)}\right)(\|\theta\|+\|\nabla \theta\|) \\
& \leq \frac{v}{2}\|\nabla \theta(t)\|^{2}+C\left(\left(1+\|w\|_{2}^{2}\right)\|\theta\|^{2}+\|\eta\|^{2}\|w\|_{2}^{2}+\|w\|_{2}^{2}\|\eta\|_{H^{-1 / 2}(\partial \Omega)}\right) .
\end{aligned}
$$

Then use kickback argument and apply estimate (4.5), Lemmas 3.2, 3.4, 3.5, 4.3 and Theorem 4.1 to arrive at

$$
\begin{aligned}
& e^{2 \alpha t}\left(\frac{v}{2}\|\nabla \theta(t)\|^{2}+2\left(c_{0}+w_{d}\right)\|\theta(t)\|_{L^{2}(\partial \Omega)}^{2}+\frac{1}{9 c_{0}}\|\theta(t)\|_{L^{4}(\partial \Omega)}^{2}+\frac{2}{3 c_{0}} \int_{\partial \Omega} w_{h}(t)^{2} \theta(t)^{2} d \Gamma\right) \\
& \quad+\int_{0}^{t} e^{2 \alpha s}\left\|\theta_{t}(s)\right\|^{2} d s \leq C h^{4}\left(\left\|w_{0}\right\|_{3}\right) \exp \left(C\left\|w_{0}\right\|_{2}\right) \\
& \quad+C \int_{0}^{t} e^{2 \alpha s}\left\|w_{t}\right\|_{2}^{2} e^{2 \alpha s}\|\nabla \theta(t)\|^{2} d s .
\end{aligned}
$$

Now a use of Gronwall's Lemma with a multiplication of $e^{-2 \alpha t}$ completes the rest of the proof.

As a consequence of Theorem 4.2, we obtain a super convergence result $\left\|\nabla\left(w_{h}(t)-\tilde{w}_{h}(t)\right)\right\|$. This, in turn, provides an optimal order of convergence result for feedback control law. Finally, the main theorem of this section, which provides optimal error estimates (optimality with respect to approximation property) in the state variable as well as feedback control law is given below.

Theorem 4.3 There is a positive constant $C=C\left(\left\|w_{0}\right\|_{3}\right)$ independent of $h$ such that

$$
\left\|w-w_{h}\right\|_{L^{\infty}\left(H^{i}\right)} \leq C h^{2-i} e^{-\alpha t} \exp \left(\left\|w_{0}\right\|_{2}\right), \quad i=0,1
$$


and

$$
\left\|v_{2 t}-v_{2 h t}\right\|_{L^{\infty}\left(L^{2}(\partial \Omega)\right)} \leq C h^{3 / 2} e^{-\alpha t} \exp \left(\left\|w_{0}\right\|_{2}\right) .
$$

Proof First part of the proof follows from estimates of $\eta$ in (4.5) and Theorems 4.1 and 4.2 with a use of triangle inequality.

For the second part, we note that

$$
v_{2 t}-v_{2 h t}=-\frac{1}{v}\left(2\left(c_{0}+w_{d}\right)(\eta-\theta)+\frac{2}{9 c_{0}}(\eta-\theta)\left(w^{2}+w w_{h}+w_{h}^{2}\right)\right) .
$$

Hence, using $w_{h}=\theta+\tilde{w}_{h}$ we get

$$
\begin{aligned}
& \left\|v_{2 t}-v_{2 h t}\right\|_{L^{\infty}\left(L^{2}(\partial \Omega)\right)} \\
& \leq C\left(\|\eta\|_{L^{\infty}\left(L^{2}(\partial \Omega)\right)}+\|\theta\|_{L^{\infty}\left(L^{2}(\partial \Omega)\right)}\right)\left(1+\|w\|_{L^{\infty}\left(L^{4}(\partial \Omega)\right)}^{2}+\left\|\tilde{w}_{h}\right\|_{L^{\infty}\left(L^{4}(\partial \Omega)\right)}^{2}+\|\theta\|_{L^{\infty}\left(L^{4}(\partial \Omega)\right)}^{2}\right) .
\end{aligned}
$$

A use of Lemmas 3.2, 4.3 and Theorem 4.2 completes the proof.

\section{Numerical Experiments}

In this section, we conduct several numerical experiments to observe stabilizability of the system (2.6)-(2.8). More precisely, the convergence of the unsteady solution to its constant steady state solution using nonlinear Neumann feedback control law are shown. Moreover, we show the order of convergence for both state variable and feedback control law by solving (2.14). Finally, our last example concerns with the stabilization of solution for the forced viscous Burgers' equation applying linear control law in which case steady state solution is nonconstant.

For a complete discrete scheme, we use a semi-implicit Characteristic-Galerkin method as follows: Let $0<k<1$ denote the time step size and $t_{n}=n k$, where $n$ is nonnegative integer. For smooth function $\phi$ defined on $[0, \infty)$, set $\phi^{n}=\phi\left(t_{n}\right)$. We now apply the CharacteristicGalerkin method to approximate

$$
\left(w_{t}, \varphi_{h}\right)+\left(w(\nabla w \cdot \mathbf{1}), \varphi_{h}\right) \approx\left(\frac{W^{n}-W^{n-1}\left(X^{n-1}(x)\right)}{k}, \varphi_{h}\right),
$$

where $X^{n-1}(x)$ is an approximation of the solution at $t=(n-1) k$ of the ordinary differential equation $\frac{d \mathbf{X}(t)}{d t}=\mathbf{W}^{n-1}(\mathbf{X}(t)), \mathbf{X}(n k)=x$, where $\mathbf{W}^{n-1}(x)=(w(x,(n-1) k), w(x,(n-$ $1) k)$ ) and $\mathbf{X}=\left(X_{1}, X_{2}\right)$. Now this can be easily solve by Freefem++ 'convect' operator command. Hence, finally we seek $\left\{W^{n}\right\}_{n \geq 1} \in V_{h}$ as a solution of

$$
\begin{aligned}
\frac{\left(W^{n}, \varphi_{h}\right)}{k} & -\frac{1}{k}\left(\left(\operatorname{convect}\left(\left[W^{n-1}, W^{n-1}\right],-k, W^{n-1}\right), \varphi_{h}\right)\right)+v\left(\nabla W^{n}, \nabla \varphi_{h}\right)+w_{d}\left(\nabla W^{n} \cdot \mathbf{1}, \varphi_{h}\right) \\
& +\left\langle 2\left(c_{0}+w_{d}\right) W^{n}+\left(\frac{2}{9 c_{0}}\left(W^{n-1}\right)^{2}\right) W^{n}, \varphi_{h}\right\rangle=0 \quad \forall \varphi_{h} \in V_{h},
\end{aligned}
$$

with $W^{0}=w_{0 h}$. For more details see [13]. For state and control trajectories final plot, we use Matlab.

Example 5.1 We choose initial condition $w_{0}$ as $w_{0}=x_{1}\left(x_{1}-1\right) x_{2}\left(x_{2}-1\right)-3$, where $w_{d}=3$ is the steady state solution and $v=1$ with $\Omega=[0,1] \times[0,1]$. For uncontrolled solution, we take zero Neumann boundary condition in (2.14) and corresponding solution is denoted as 


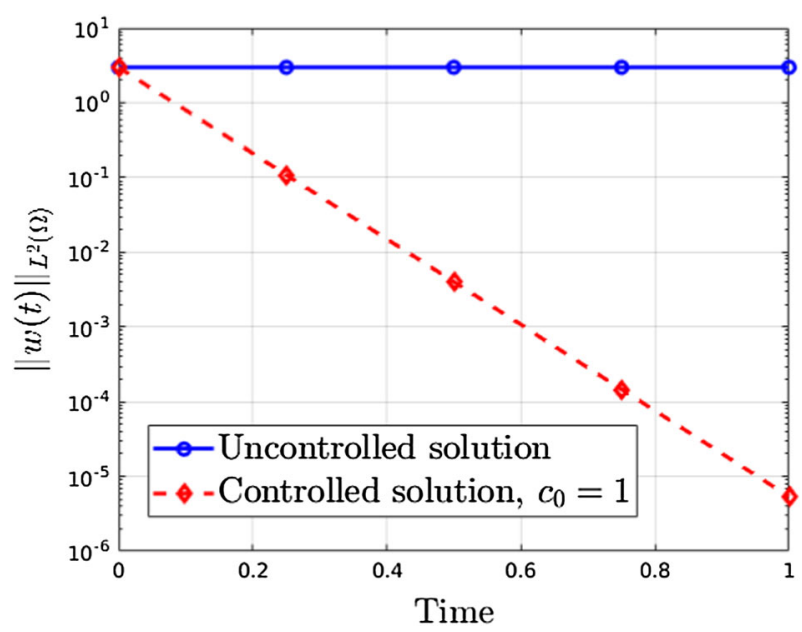

Fig. 1 State, Example 5.1

Table 1 Errors and convergence rate of $w$ when $c_{0}=1, k=0.0001$ and $t=1$ for Example 5.1

\begin{tabular}{lllll}
\hline$h$ & $\left\|w\left(t_{n}\right)-W^{n}\right\|$ & Conv. rate & $\left\|w\left(t_{n}\right)-W^{n}\right\|_{1}$ & Conv. rate \\
\hline$\frac{1}{4}$ & $1.26 \times 10^{-7}$ & & $5.82 \times 10^{-7}$ & \\
$\frac{1}{8}$ & $3.82 \times 10^{-8}$ & 1.72 & $2.43 \times 10^{-7}$ & 1.26 \\
$\frac{1}{16}$ & $9.78 \times 10^{-9}$ & 1.96 & $1.07 \times 10^{-7}$ & 1.18 \\
$\frac{1}{32}$ & $2.44 \times 10^{-9}$ & 2.01 & $4.88 \times 10^{-8}$ & 1.12 \\
$\frac{1}{64}$ & $5.99 \times 10^{-10}$ & 2.03 & $2.13 \times 10^{-8}$ & 1.19 \\
\hline
\end{tabular}

Table 2 Errors and convergence rate of $v_{2}$ when $c_{0}=1$ and $t=1$ for Example 5.1

\begin{tabular}{lll}
\hline$h$ & $\left|v_{2}\left(t_{n}\right)-v_{2 h}\left(t_{n}\right)\right|$ & Conv. rate \\
\hline$\frac{1}{4}$ & $8.75 \times 10^{-7}$ & \\
$\frac{1}{8}$ & $2.61 \times 10^{-7}$ & 1.74 \\
$\frac{1}{16}$ & $6.67 \times 10^{-8}$ & 1.97 \\
$\frac{1}{32}$ & $1.65 \times 10^{-8}$ & 2.01 \\
$\frac{1}{64}$ & $4.03 \times 10^{-9}$ & 2.04 \\
\hline
\end{tabular}

"Uncontrolled solution" in Fig. 1. For controlled solution, we choose the Neumann control (2.13) with $c_{0}=1$ and corresponding solution is denoted as "Controlled solution, $c_{0}=1$ " in Fig. 1.

From Fig. 1, we can easily see that without any control i.e. with zero Neumann boundary, solution of (2.14) does not settle at zero, whereas applying the control (2.13), the solution for the problem (2.14) in $L^{2}$ - norm goes to zero. Also it is observed that for other values of $c_{0}>0$, the system (2.14) is stabilizable. From Table 1, it follows that $L^{2}$ and $H^{1}$ orders of convergence for state variable $w(t)$ are 2 and 1 , respectively, which confirms our theoretical results established in Theorem 4.3. Since the exact solution is unknown in this case, we have taken very refined mesh solution as exact solution to compute the order of convergence. In 


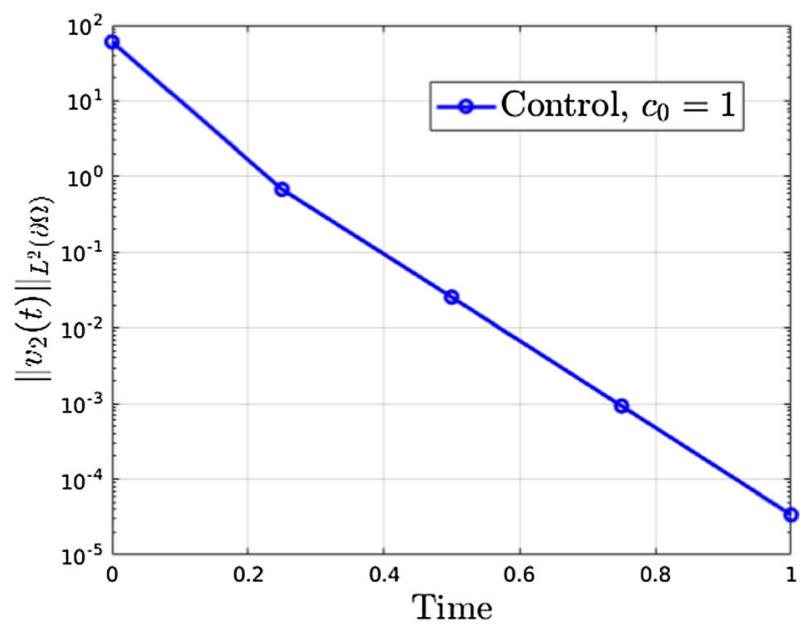

Fig. 2 Control, Example 5.1

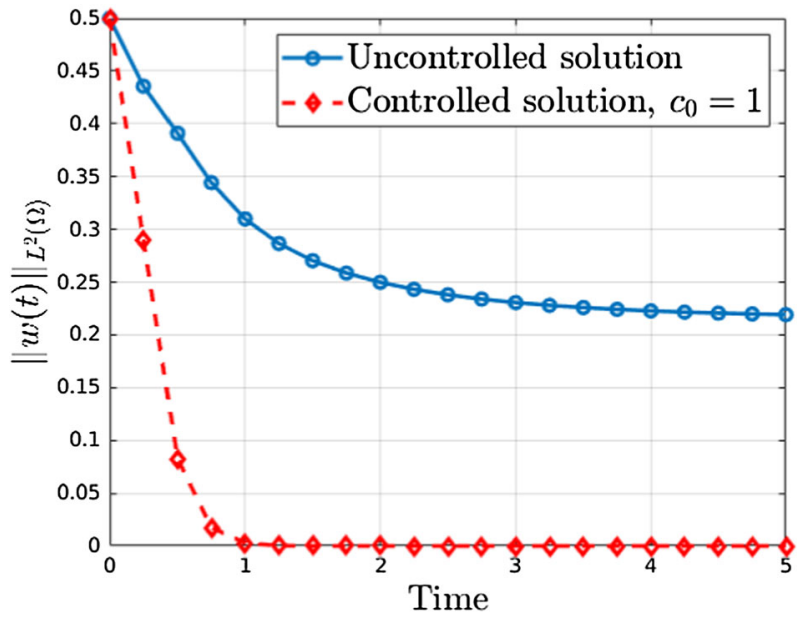

Fig. 3 State, Example 5.2

Table 2, it is noted that the order of convergence of feedback control law (2.13) is 2, while theoretically it is proved to be $3 / 2$ in Theorem 4.3 . Behavior of the trajectory of the control law (2.13) which tends to zero, is shown in Fig. 2.

Example 5.2 In this example, take the initial condition $w_{0}=\sin \left(\pi x_{1}\right) \sin \left(\pi x_{2}\right)$ and $v=0.05$, $c_{0}=1$ with 0 as the steady state solution in $\Omega=[0,1] \times[0,1]$.

From Fig. 3, it is observed that steady state solution $w_{d}=0$ is unstable in the first case denoted as "Uncontrolled solution". But using the control law (2.13), it is shown that state $w$ in $L^{2}$ - norm goes to zero exponentially. Figure 4 indicates how control law (2.13) behave with time and tends to settle at zero after some time.

Now the following example is related to the Remark 3.2 where the control law is applied to some part of the boundary. In the remaining part, either zero Dirichlet or zero Neumann condition is considered. 


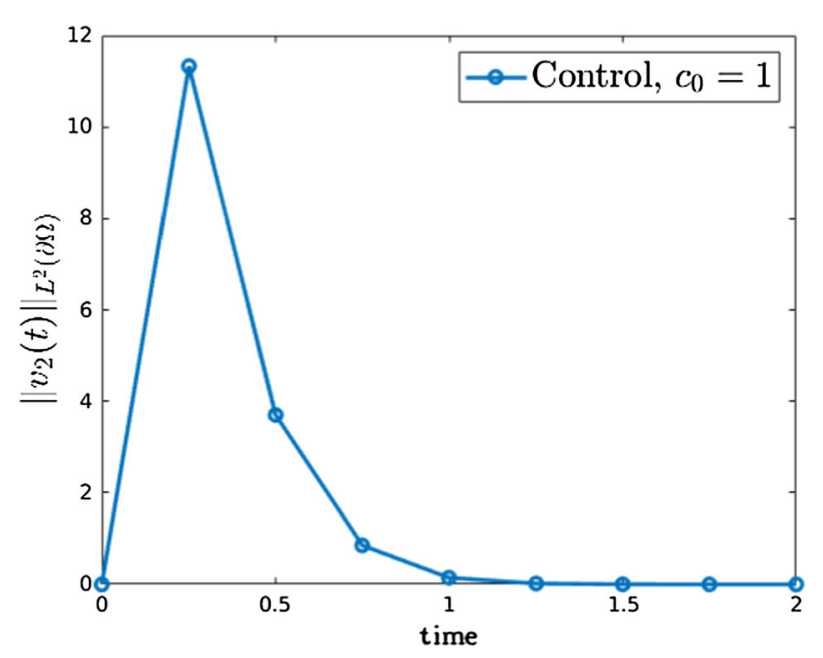

Fig. 4 Control, Example 5.2

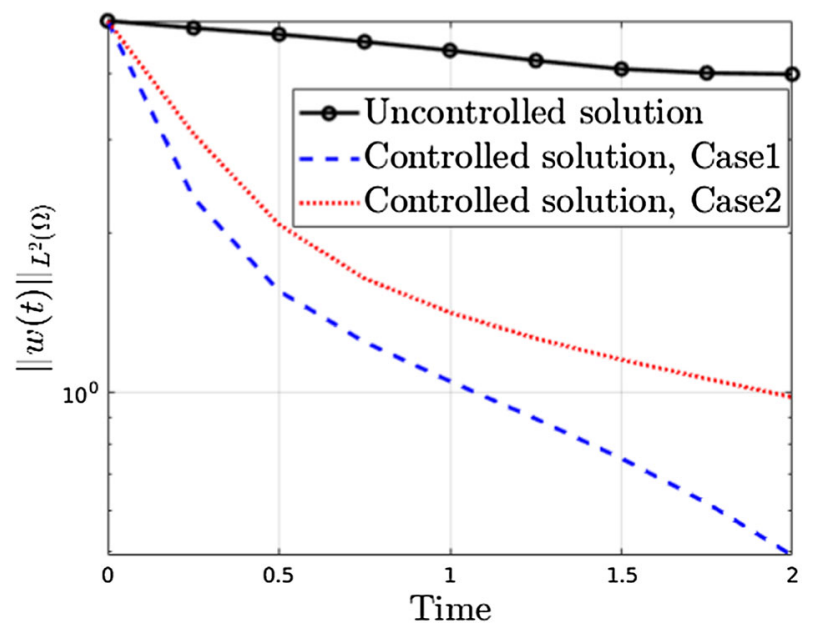

Fig. 5 State, Example 5.3

Example 5.3 We take the initial condition $w_{0}=\cos \left(\pi x_{1}\right) \cos \left(\pi x_{2}\right)-5$, where $w_{d}=5$ is the steady state solution with $v=0.01$ and $\Omega=[0,1] \times[0,1]$. We consider two cases.

Case 1 Take zero Dirichlet boundary $\Gamma_{D}=1 \times[0,1]$ and on the remaining parts Neumann boundary control $\Gamma_{N}$. For controlled solution, we take (2.13) with $c_{0}=10$ on $\Gamma_{N}$ i.e. on 3 parts of the boundary. We then compare the trajectories with another case which is given below.

Case 2 We consider zero Dirichlet boundary $\Gamma_{D}=1 \times[0,1]$, put the Neumann boundary control on $0 \times[0,1]$ and put zero Neumann boundary condition on other 2 parts. So, in Case 2 control works only on one part of the boundary.

For uncontrolled solution, we take zero Neumann boundary condition on $\Gamma_{N}$ and zero Dirichlet on $\Gamma_{D}=1 \times[0,1]$. 


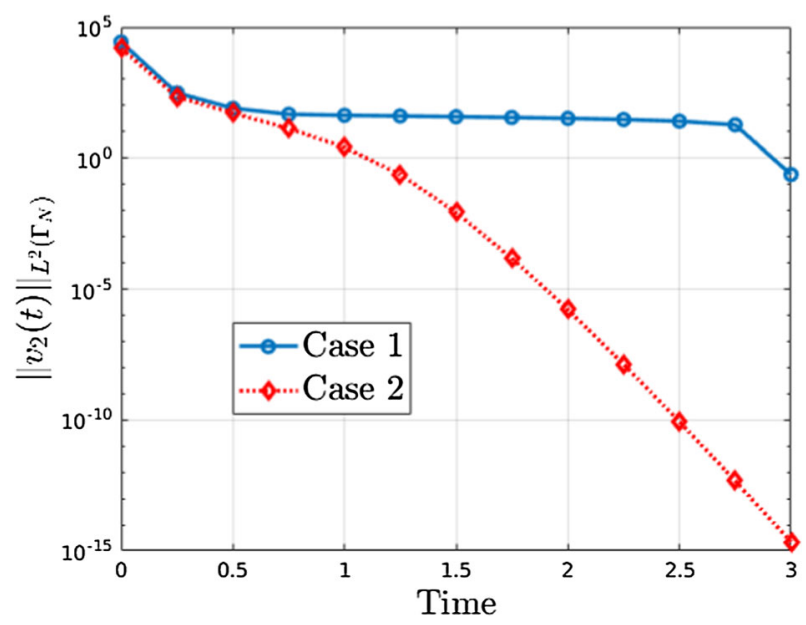

Fig. 6 Control, Example 5.3

From Fig. 5, it is clear that with homogeneous mixed boundary condition, uncontrolled solution does not change its state to zero whereas in presence of control on $\Gamma_{N}$ only, state and control trajectories go to zero both in Case 1 and Case 2. Since in Case 2, control works only in one part of the boundary, so control needs more time compare to Case 1 to settle its state to zero, which is also visible in Fig. 5. The $L^{2}$ - norm of feedback control has a tendency to settle at zero faster in the Case 2 compare to Case 1 which is documented in Fig. 6. Also we have not observed much differences in the trajectories if we change the zero Dirichlet by zero Neumann condition in Case 1 . Concerning zero Dirichlet boundary on more parts of the boundary e.g. if we take $w=0$ on 3 parts of the boundary with Neumann control on remaining one part, corresponding state tends to settle at zero even within $t<1$ since with zero Dirichlet boundary, the system is already stable.

Below we discuss another example, where steady state solution is not a constant for the forced Burgers' equation. It will be shown that, even with the linear control law, system can be stabilizable numerically.

Example 5.4 We now consider a case when the steady state solution is not constant:

$$
\begin{aligned}
& u_{t}-v \Delta u+u(\nabla u \cdot \mathbf{1})=f^{\infty} \quad \text { in } \quad(x, t) \in \Omega \times(0, \infty), \\
& \frac{\partial u}{\partial n}(x, t)=g^{\infty}+v_{2}(x, t) \quad \text { on } \quad(x, t) \in \partial \Omega \times(0, \infty), \\
& u(x, 0)=u_{0}(x) \quad x \in \Omega,
\end{aligned}
$$

where, $f^{\infty}$ and $g^{\infty}$, independent of $t$ are functions of $x_{1}$ and $x_{2}$ only. Corresponding equilibrium or steady state solution $u^{\infty}$ of the unsteady state problem satisfies

$$
\begin{aligned}
-v \Delta u^{\infty}+u^{\infty}\left(\nabla u^{\infty} \cdot \mathbf{1}\right) & =f^{\infty} \quad \text { in } \quad \Omega, \\
\frac{\partial u^{\infty}}{\partial n} & =g^{\infty} \quad \text { on } \quad \partial \Omega .
\end{aligned}
$$


Fig. 7 State, Example 5.4
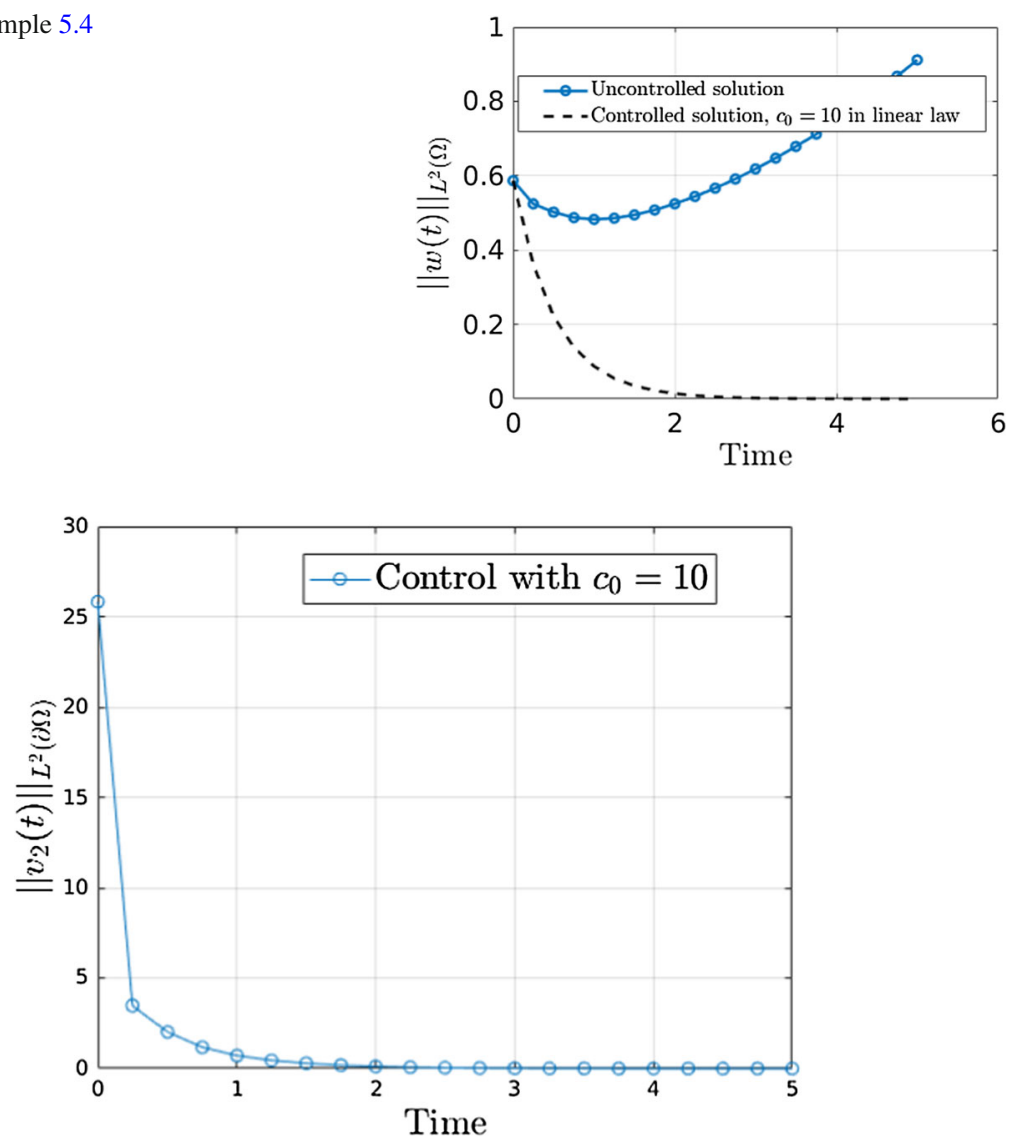

Fig. 8 Control, Example 5.4

Let $w=u-u^{\infty}$. Then, $w$ satisfies

$$
\begin{aligned}
& w_{t}-v \Delta w+u^{\infty}(\nabla w \cdot \mathbf{1})+w\left(\nabla u^{\infty} \cdot \mathbf{1}\right)+w(\nabla w \cdot \mathbf{1})=0 \quad \text { in } \quad(x, t) \in \Omega \times(0, \infty), \\
& \frac{\partial w}{\partial n}(., t)=v_{2}(x, t), \quad \text { on } \partial \Omega \times(0, \infty), \\
& w(0)=u_{0}-u^{\infty}=w_{0} \text { (say) in } \Omega .
\end{aligned}
$$

Similarly as before we solve (5.4) using Freefem++ as in (5.1) with linear control law $v_{2}=-\frac{1}{v} c_{0} W^{n}$.

For the numerical experiment, we choose viscosity parameter $v=0.1$, steady state solution $u^{\infty}=-0.2 x_{1}$, forcing function $f^{\infty}=0.04 x_{1}$ and $g^{\infty}=-0.2 n_{1}$, control parameter $c_{0}=10$ with initial condition $w_{0}=\sin \left(\pi x_{1}\right) \sin \left(\pi x_{2}\right)+0.2 x_{1}$ in $\Omega=[0,1] \times[0,1]$.

From the first draw line in Fig. 7, we observe that nonconstant steady state solution is not asymptotically stable with zero Neumann boundary condition. But, using the linear control law $-\frac{1}{v} c_{0} W^{n}$, system (5.4) is stabilizable which is also documented in Fig. 7. Figure 8 shows that the linear control law $-\frac{1}{v} c_{0} W^{n}$ decays to zero as time increases. However, we do not 
have a theoretical result to substantiate this observation. We believe that the system is locally stabilizable with this linear control law.

\section{Concluding Remarks}

In this paper, global stabilization results for the two dimensional viscous Burgers' equation are established in $L^{\infty}\left(H^{i}\right), i=0,1,2$ norms, when the steady state solution is constant. Optimal error estimates in $L^{\infty}\left(L^{2}\right)$ and in $L^{\infty}\left(H^{1}\right)$ for the state variable are established. Further, error estimate for the feedback controller is also shown. All the results are verified by numerical examples. Now under addition of forcing function in the two dimensional viscous Burgers' equation, the steady state solution is no more constant and as such the present analysis does not hold for nonconstant steady state case. Hence, the analysis for two dimensional generalized forced viscous Burgers' equation will be addressed in future.

Acknowledgements Both authors acknowledge the valuable suggestions and comments given by honorable referees which help to improve the manuscript. The first author was supported by the ERC advanced Grant 668998 (OCLOC) under the EUs H2020 research program.

Funding Open access funding provided by University of Graz.

Open Access This article is licensed under a Creative Commons Attribution 4.0 International License, which permits use, sharing, adaptation, distribution and reproduction in any medium or format, as long as you give appropriate credit to the original author(s) and the source, provide a link to the Creative Commons licence, and indicate if changes were made. The images or other third party material in this article are included in the article's Creative Commons licence, unless indicated otherwise in a credit line to the material. If material is not included in the article's Creative Commons licence and your intended use is not permitted by statutory regulation or exceeds the permitted use, you will need to obtain permission directly from the copyright holder. To view a copy of this licence, visit http://creativecommons.org/licenses/by/4.0/.

\section{References}

1. Adams, R.A., Fournier, J.J.F.: Sobolev Spaces. Elsevier, Amsterdam (2003)

2. Agmon, S.: Lectures on Elliptic Boundary Value Problems. AMS Chelsea Publishing, Providence (2010)

3. Balogh, A., Krstic, M.: Burgers' equation with nonlinear boundary feedback: $H^{1}$ stability well-posedness and simulation. Math. Probl. Eng. 6, 189-200 (2000)

4. Buchot, J.M., Raymond, J.P., Tiago, J.: Coupling estimation and control for a two dimensional Burgers type equation. ESAIM Control Optim. Calc. Var. 21, 535-560 (2015)

5. Burns, J.A., Kang, S.: A control problem for Burgers' equation with bounded input/output. Nonlinear Dyn. 2, 235-262 (1991)

6. Burns, J.A., Kang, S.: A stabilization problem for Burgers' equation with unbounded control and observation. vol. 8-14 (1990)

7. Byrnes, C.I., Gilliam, D.S., Shubov, V.I.: On the global dynamics of a controlled viscous Burgers' equation. J. Dyn. Control Syst. 4, 457-519 (1998)

8. Cannon, J.R., Ewing, R.E., He, Y., Lin, Y.-P.: A modified nonlinear Galerkin method for the viscoelastic fluid motion equations. Int. J. Eng. Sci. 37, 1643-1662 (1999)

9. Ciarlet, P.G.: The Finite Element Method for Elliptic Problems, Studies in Applied Mathematics and Its Applications, vol. 4. North-Holland Publishing Co., Amsterdam (1978)

10. Douglas, J., Dupont, T.: Galerkin methods for parabolic equations with nonlinear boundary conditions. Numer. Math. 20, 213-237 (1973)

11. Goswami, D., Pani, A.K.: A priori error estimates for semidiscrete finite element approximations to the equations of motion arising in Oldroyd fluids of order one. Int. J. Numer. Anal. Model. 8, 324-352 (2011)

12. He, Y., Lin, Y., Shen, S., Tait, R.: On the convergence of viscoelastic fluid flows to a steady state. Adv. Differ. Equ. 7, 717-742 (2002) 
13. Hecht, F.: Freefem++, Version 3.58-1, Laboratoire Jacques-Louis Lions, Université Pierre et Marie Curie, Paris

14. Heywood, J.G., Rannacher, R.: Finite element approximation of the nonstationary Navier-Stokes problem: I. Regularity of solutions and second order error estimates for spatial discretization. SIAM J. Numer. Anal. 19, 275-311 (1982)

15. Hinze, M., Volkwein, S.: Analysis of instantaneous control for the Burgers equation. Nonlinear Anal. Theor. 50, 1-26 (2002)

16. Ito, K., Kang, S.: A dissipative feedback control for systems arising in fluid dynamics. SIAM J. Control Optim. 32, 831-854 (1994)

17. Ito, K., Yan, Y.: Viscous scalar conservation laws with nonlinear flux feedback and global attractors. J. Math. Anal. Appl. 227, 271-299 (1998)

18. Krstic, M.: On global stabilization of Burgers' equation by boundary control. Syst. Control Lett. 37, 123-141 (1999)

19. Kundu, S., Pani, A.K.: Finite element approximation to global stabilization of the Burgers' equation by Neumann boundary feedback control law. Adv. Comput. Math. 44, 541-570 (2018)

20. Kundu, S., Pani, A.K.: Global stabilization of BBM-Burgers' type equations by nonlinear boundary feedback control laws: theory and finite element error analysis. J. Sci. Comput. 81, 845-880 (2019)

21. Lions, J.L., Magenes, E.: Problèmes aux Limites Non Homogènes et Applications. Dunod, Paris (1968)

22. Liu, W.J., Krstic, M.: Adaptive control of Burgers equation with unknown viscosity. Int. J. Adapt. Control Signal Process 15, 745-766 (2001)

23. Ly, H.V., Mease, K.D., Titi, E.S.: Distributed and boundary control of the viscous Burgers' equation. Numer. Funct. Anal. Optim. 18, 143-188 (1997)

24. Nirenberg, L.: On elliptic partial differential equations. Ann. Scuola Norm. Sup. Pisa 3(13), 115-162 (1959)

25. Raymond, J.P.: Feedback boundary stabilization of the two-dimensional Navier-Stokes equations. SIAM J. Control Optim. 45, 790-828 (2006)

26. Smaoui, N.: Nonlinear boundary control of the generalized Burgers equation. Nonlinear Dyn. 37, 75-86 (2004)

27. Smaoui, N.: Boundary and distributed control of the viscous Burgers equation. J. Comput. Appl. Math. 182, 91-104 (2005)

28. Sobolevskii, P.E.: Stabilization of viscoelastic fluid motion (Oldroyd's mathematical model). Differ. Integral Equ. 7, 1597-1612 (1994)

29. Thevenet, L., Buchot, J.M., Raymond, J.P.: Nonlinear feedback stabilization of a two-dimensional Burgers' equation. ESAIM Control Optim. Calc. Var. 16, 929-955 (2010)

30. Temam, R.: Navier-Stokes Equations, Theory and Numerical Analysis. North-Holland, Amsterdam (2002)

31. Thomee, V.: Galerkin Finite Element Methods for Parabolic Problems. Springer, Berlin (1997)

Publisher's Note Springer Nature remains neutral with regard to jurisdictional claims in published maps and institutional affiliations. 